\title{
Trajectory Design of Perseus: A CubeSat Mission Concept to Phobos
}

\author{
Ravi teja Nallapu ${ }^{1,+}+$ (i) Graham Dektor ${ }^{2,+}$, Nalik Kenia ${ }^{2,+}$, James Uglietta ${ }^{2,+}$, Shota Ichikawa ${ }^{2,+}$, \\ Mercedes Herreras-Martinez ${ }^{2,+}$, Akshay Choudhari ${ }^{2, \dagger}$, Aman Chandra ${ }^{1, \dagger}$, Stephen Schwartz ${ }^{3,+}(\mathbb{0}$, \\ Erik Asphaug ${ }^{3}$ and Jekanthan Thangavelautham ${ }^{1, *}$ iD \\ 1 Department of Aerospace and Mechanical Engineering, University of Arizona, Tucson, AZ 85721, USA; \\ rnallapu@email.arizona.edu (R.t.N.); achandra@email.arizona.edu (A.C.) \\ 2 School for Engineering of Matter, Arizona State University, Tempe, AZ 85287, USA; gdektor@asu.edu (G.D.); \\ nkenia@gmail.com (N.K.); jugliett@asu.edu (J.U.); shota4711@gmail.com (S.I.); \\ mherre15@asu.edu (M.H.-M.); aachoud1@asu.edu (A.C.) \\ 3 Lunar and Planetary Laboratory, University of Arizona, Tucson, AZ 85721, USA; \\ srs51@email.arizona.edu (S.S.); asphaug@lpl.arizona.edu (E.A.) \\ * Correspondence: jekan@arizona.edu; Tel.: +1-520-621-9638 \\ + These authors contributed equally to this work.
}

Received: 20 October 2020; Accepted: 4 December 2020; Published: 15 December 2020

\begin{abstract}
The Martian satellites Phobos and Deimos hold many unanswered questions that may provide clues to the origin of Mars. These moons are low $\Delta v$ stopover sites to Mars. Some human missions to Mars typically identify Phobos and Deimos as staging bases for Mars surface exploration. Astronauts could base initial operations there in lieu of repeated voyages to and from the planet surface, to refuel transiting spacecraft, to teleoperate robotics and other critical machinery, and to develop habitable infrastructure ahead of human landings. Despite their strategic and scientific significance, there has been no successful dedicated mission to either moon. For this reason, we propose Perseus, a geological imaging CubeSat mission to Phobos. Perseus, a 27U, $54 \mathrm{~kg}$ CubeSat will return thermal and visible images at resolutions better than currently available over most of Phobos' surface. This includes visible images at $5 \mathrm{~m} /$ pixel and thermal images at $25 \mathrm{~m} /$ pixel of Phobos' surface. The Perseus mission is nominally intended to be a co-orbital mission, where the spacecraft will encounter Phobos on its Martian orbit. However, a hyperbolic rendezvous mission concept, to image Phobos on a hyperbolic flyby, is also considered to reduce the risks associated with orbit capture and to reduce mission costs. This paper presents the preliminary feasibility, science objectives, and technological development challenges of achieving these science goals. We then formulate two rendezvous concepts as a series of three nonlinear optimization problems that span the design tree of mission concepts. The tree's root node is the heliocentric cruise problem, which identifies the near-optimal launch and arrival windows for the Perseus spacecraft. The leaf nodes of the design tree are the two rendezvous concepts that identify near-optimal co-orbital and hyperbolic trajectories for Phobos' reconnaissance. The design problems are solved using evolutionary algorithms, and the performance of the selected mission concepts is then examined. The results indicate that a co-orbital encounter allows about one encounter per day with about $6 \mathrm{~min}$ per encounter. The hyperbolic encounter, on the other hand, allows a single encounter where the spacecraft will spend about $2 \mathrm{~min}$ in the imaging region with respect to Phobos. The spacecraft will obtain higher resolution images of Phobos on this feasible region than have ever been seen for most of the surface. These detailed images will help identify candidate landing sites and provide critical data to derisk future surface missions to Phobos.
\end{abstract}


Keywords: planetary moon exploration mission; interplanetary mission design; CubeSat systems engineering; co-orbital reconnaissance; hyperbolic reconnaissance

\section{Introduction}

CubeSats have emerged as low-cost options for interplanetary exploration. Thanks to the miniaturization of electronics, power systems, instruments, and propulsion systems, it is possible to develop a small spacecraft that operates autonomously in deep space. Deep space CubeSats are being developed to explore the Moon, Mars, and near-Earth asteroids [1]. NASA JPL's MarCO mission was a pair of $6 \mathrm{U}$ CubeSats that were launched alongside NASA's INSIGHT mission to Mars. They have paved the way for a whole new class of interplanetary missions [2,3]. The CubeSats traveled alongside INSIGHT, and, upon reaching Mars, acted as a communication relay, transmitting data from INSIGHT lander to the DSN during Entry, Descent, and Landing (EDL) maneuvers.

Inspired by the capabilities of MarCO, we formulate a science-focused mission concept for a 27U CubeSat heading towards Mars, with Phobos, the innermost moon (semi-major axis 2.76 Mars radii and spiraling inward [4] as the science target. If successful, such as mission concept could be a template to develop low-cost, focused, small-satellite missions to explore minor-moons. We describe Perseus, a 27U CubeSat mission concept that shall be deployed similar to MarCO from an Earth-escaping rocket on a trajectory to Mars and where Earth and Mars are at closes approach. These opportunities to Mars appear every two years. Our overall concept is to utilize many of the critical subsystems demonstrated on MarCO, but added with it science instruments to perform reconnaissance science. Unlike MarCO, Perseus shall contain a propulsion system that will enable it to perform complex maneuvers, including achieving a Martian orbit. We compare this against a simple one-time flyby of Phobos. Utilizing a low-cost CubeSat to perform reconnaissance science makes practical sense as there has been high number of losses of spacecraft to Mars and Phobos. It makes sense to spread the risk to smaller low-cost missions so that even a loss can be quickly recovered with another reflight. In addition, this science reconnaissance mission can provide high resolution surface images of Phobos not possible with current assets including HiRise on MRO that can only see the tidally locked side of Phobos. This high resolution images can provide better insight into what are theorized to be dynamical processes on the surface of Phobos, including movement of dust grains and movement striations, shed light into Phobos' origins, identify local resources and identify landing sites for future mission and human transit bases. Using this low-cost CubeSat, we can choose to get into very close encounter flybys with Phobos after the primary mission that might be too risky for a flagship mission. Furthermore, this mission would serve as template to explore other minor moons in the solar system through flyby co-orbits. Once the spacecraft has achieved a capture orbit around Mars, it shall maneuver into a co-orbit with Phobos. The proposed mission is to be undertaken by the University of Arizona's Space and Terrestrial Robotic Exploration Laboratory, in collaboration with NASA JPL and shall be utilizing mostly Commercial-Off-The-Shelf (COTS) components. We expect the spacecraft to be built over three years and compete for a mission opportunity through NASA's SIMPLEX program. The primary mission requirement of Perseus is to capture into Mars orbit and attain low-altitude flyovers of Phobos. The spacecraft shall return high-quality science data from these flyovers. The science requirements (Table 1) are defined by two fundamental science knowledge gaps: the need for high-resolution visible and thermal images of the surface Phobos. Specifically, we require thermal and visible images of more than half the Phobos' surface, at a resolution greater than what is currently available, obtaining better and more homogeneous coverage than what currently exists. The spacecraft shall have opportunities to get higher resolutions images of peculiar formations and boulders on Phobos' surface, such as the $90 \mathrm{~m}$ rock formation popularized by former astronaut Buzz Aldrin as the "monolith." 
Table 1. Science traceability matrix for the Perseus mission concept.

\begin{tabular}{|c|c|c|}
\hline $\begin{array}{l}\text { Perseus } \\
\text { Science Objectives }\end{array}$ & $\begin{array}{l}\text { Geophysics } \\
\text { Return thermal images of more than half the } \\
\text { surface of Phobos, at a resolution greater than what is } \\
\text { currently available. }\end{array}$ & $\begin{array}{l}\text { Evolution } \\
\text { Return visible images of more than half the surface of } \\
\text { Phobos, at a resolution greater than what is currently } \\
\text { available. }\end{array}$ \\
\hline $\begin{array}{l}\text { Physical } \\
\text { Parameters }\end{array}$ & $\begin{array}{l}\text { 1. Surface composition } \\
\text { 2. Surface grain size } \\
\text { 3. Ponding of regolith } \\
\text { 4. Presence of big boulders }\end{array}$ & $\begin{array}{l}\text { 1. 3D topography and shape } \\
\text { 2. Steep surface slopes } \\
\text { 3. Degradation of craters and striations by subsequent } \\
\text { events } \\
\text { 4. Ponding of regolith } \\
\text { Presence of big boulders } \\
\text { 5. Boulder size frequency } \\
\text { Surface albedo, texture and distribution } \\
\text { 6. Superposition of striations and crater intersections } \\
\text { 7. Degradation of striations }\end{array}$ \\
\hline Observables & $\begin{array}{l}\text { Temperature of surface features at different times } \\
\text { of the day. Evident surface shape and texture. }\end{array}$ & $\begin{array}{l}\text { Surface shape, texture and visible photometric } \\
\text { response at the spatial scale ( } 100 \mathrm{~m}) \text { of a typical } \\
\text { striation. }\end{array}$ \\
\hline $\begin{array}{l}\text { Data } \\
\text { Requirements }\end{array}$ & $\begin{array}{l}\text { 1. } 3 \text { distinct temperature measurements at different } \\
\text { day times. } \\
\text { 2. } 50 \% \text { surface coverage. } \\
\text { 3. Temperature accuracy }<3 \mathrm{~K} \text {. }\end{array}$ & $\begin{array}{l}\text { 1. Greyscale images }>10 \times \text { resolution } \\
\text { than average striation width } \\
\text { 2. More than } 50 \% \text { of surface coverage } \\
\text { 3. Greyscale images overlapping thermal } \\
\text { imager coverage. }\end{array}$ \\
\hline $\begin{array}{l}\text { Instrument } \\
\text { Performance } \\
\text { Requirements }\end{array}$ & $\begin{array}{l}\text { TC: Thermal Camera } \\
\text { 1. NETD }<50 \mathrm{mK} @ \text { operating temperature } \\
\text { 2. Spatial Resolution }<100 \mathrm{~m} / \text { pixel } \\
\text { 3. Integration time } \leq 5 \mathrm{~s}\end{array}$ & $\begin{array}{l}\text { VSC: Visual Science Camera } \\
\text { 1. Spatial resolution }<10 \mathrm{~m} / \text { pixel } \\
\text { 2. } 10 \text {-bit RAW monochrome } \\
\text { 3. SNR }>35 \mathrm{~dB} \\
\text { 4. Integration time } \leq 1 \mathrm{~s}\end{array}$ \\
\hline $\begin{array}{l}\text { Projected } \\
\text { Instrument } \\
\text { Performance }\end{array}$ & $\begin{array}{l}\text { 1. Temperature accuracy: } 50 \mathrm{mK} \\
\text { 2. SR@ } 50 \mathrm{~km}: 25 \mathrm{~m} / \text { pixel } \\
\text { 3. NETD }<50 \mathrm{mK} \\
\text { 4. AFoV: } 18^{\circ} \times 14^{\circ} \\
\text { 5. Operating temperature } 233-353 \mathrm{~K} \\
\text { 6. Integration time } \leq 5 \mathrm{~s}\end{array}$ & $\begin{array}{l}\text { 1. SR @ } 50 \mathrm{~km}: 5.3 \mathrm{~m} / \text { pixel } \\
\text { 2. Max Frame Rate: } 60 \mathrm{fps} \\
\text { 3. SNR: } 39 \mathrm{~dB} \\
\text { 4. QE: } 60 \% \\
\text { 5. DNR: } 65 \mathrm{~dB} \\
\text { 6. AFoV: } 7.8^{\circ} \times 6.2^{\circ} \\
\text { 7. Operating temperature } 233-333 \mathrm{~K}\end{array}$ \\
\hline $\begin{array}{l}\text { Mission } \\
\text { Requirements }\end{array}$ & $\begin{array}{l}\text { 1. Capture into Mars orbit } \\
\text { 10+ flybys within } 100 \mathrm{~km} \text { of Phobos } \\
\text { 2. Pointing accuracy of }<1^{\circ} \\
\text { Slew rate of }>1 \mathrm{deg} / \mathrm{s} \\
\text { 3. A priori position knowledge relative to Phobos } \\
\text { better than } 1 \mathrm{~km} \\
\text { 4. Return } 40 \mathrm{MB} \text { data } \\
\text { 5. Ambient temp }<353 \mathrm{~K} \\
\text { 6. Observations at }>1^{\circ} \text { sun incidence }\end{array}$ & $\begin{array}{l}\text { 1. Capture into Mars orbit } \\
\text { 2. } 10+\text { flybys within } 100 \mathrm{~km} \text { of Phobos } \\
\text { 3. Pointing accuracy of }<1^{\circ} \\
\text { 4. A priori position knowledge relative to Phobos } \\
\text { better than } 1 \mathrm{~km} \\
\text { 5. Return } 370 \mathrm{MB} \text { data } \\
\text { 6. Ambient temp }<333 \mathrm{~K} \\
\text { 7. Observations at }>1^{\circ} \text { sun incidence }\end{array}$ \\
\hline $\begin{array}{l}\text { SNR: } \\
\text { Signal to Noise Ratio }\end{array}$ & $\begin{array}{l}\text { DNR: } \\
\text { Dynamic Range }\end{array}$ & $\begin{array}{l}\text { SR: } \\
\text { Spatial Resolution }\end{array}$ \\
\hline $\begin{array}{l}\text { QE: } \\
\text { Quantum Efficiency }\end{array}$ & $\begin{array}{l}\text { AFoV: } \\
\text { Angular Field of View }\end{array}$ & $\begin{array}{l}\text { NETD: } \\
\text { Noise Equivalent Temperature Difference }\end{array}$ \\
\hline
\end{tabular}

Perseus shall, for the first time, demonstrate a CubeSat achieving a capture orbit around Mars. The spacecraft, similar to India's Mangalayan orbiter, shall use a single impulsive burn at the closest approach to enter into a highly elliptical capture orbit that minimizes injection $\Delta v$. The CubeSat shall then maneuver to perform aeroassist over several months and match its apoapsis to that of Phobos. Science operations commence during repeated low-velocity flybys within $100 \mathrm{~km}$ of the satellite and continue until the end of the science mission.

The CubeSat shall then enter a disposal orbit that meets current Planetary Protection standards. We review previously attempted missions to Phobos in Section 2, followed by a description of the science goals in Section 3. Section 4 describes the concept of operations of the Perseus mission and Section 5 describes the baseline spacecraft subsystems populated from existing commercial spacecraft hardware. Section 6 presents the trajectory design of the Perseus spacecraft as a tree of design problems. These problems are solved in Section 7 using Evolutionary Algorithms. Finally, Section 8 concludes the current work by summarizing the key points of this paper and identifying the next steps in advancing the Perseus mission. 


\section{Related Work}

The earliest interplanetary missions beyond the Moon focused on the enigmatic neighboring planets, Mars and Venus. Venus has no satellites, while Mars has two small moons, tens of kilometers in diameter, with a surface gravity of about $1 / 1000$ that of Earth. Phobos and Deimos were photographed by some of the early Mars probes as flyby targets of opportunity, beginning with Mariner 7 in 1969, which was a Mars flyby to study the surface and atmosphere of Mars [5]. In 1971, Mariner 9 became the first spacecraft to orbit another planet, capturing the first close-up images of Phobos and a total of 214 images of Phobos and Deimos, some as close as $1200 \mathrm{~km}$ with the smallest pixel scale of $30 \mathrm{~m}$ [6]. The era of small-body planetary geology had begun.

The next missions to explore Phobos were the Viking missions that were launched in 1976 and placed two orbiters into Mars orbit and two landers on the Martian surface. The Viking mission made several discoveries of Phobos, including finding a global network of grooves, the first mass determination, and the surface features [6]. Those discoveries directed people's interest in Phobos and opened the pathway to a dedicated Phobos mission. There have been three missions dedicated to studying Phobos, which includes the twin spacecraft Phobos 1 and Phobos 2 and the spacecraft Phobos-Grunt. These were ambitious lander missions with sample return that included geophysical and compositional probing of the small moon's surface. Phobos 1 was lost on its way to Mars, and Phobos 2 lost contact nearly an hour before it was due for a touch-down on Phobos. Despite not achieving its primary mission objectives, Phobos 2 obtained meaningful and unique scientific data of Phobos and its space environment, including visible and near-IR imaging and thermal and spectral properties of the Phobos regolith from distances of 190-1100 km [6]. Most recently, Phobos-Grunt was a follow-up to Phobos 2 but was lost before attaining Earth escape. Data obtained from these various Mars and Phobos missions raised many more questions than answers, and the challenges of getting to Phobos reminds us of the importance of a multi-pronged exploration strategy, in particular a blend of medium- to high-cost missions, along with much smaller targeted reconnaissance missions such as Perseus that can attain some of the same science goals.

Missions to Mars have obtained some of the most valuable data of Phobos to date [7]. Mars Pathfinder Rover made multispectral measurements of Phobos in 1996, and in the next year, 1997, Mars Global Surveyor (MGS) obtained Mars Orbiter Camera data, Thermal Emission Spectrometer data, and Mars Orbiter Later Altimeter data of Phobos during its aerobraking phase. Mars Express (MEx) arrived in 2003 and inserted into an orbit that makes repeated Phobos-orbit crossings; it has since obtained the most detailed science data of Phobos yet. Although the primary objectives of MEx were the orbital study of the interior, subsurface, surface, atmosphere, and environment of Mars, it has made numerous passes within $3000 \mathrm{~km}$ of Phobos during its mission lifetime. Those investigations provided astrometric data, the first high-resolution global map, and some near-IR mineralogical mapping of Phobos. MEx is still performing its extended mission.

Mars Reconnaissance Orbiter (MRO) is a spacecraft designed and launched in 2006 to study the geologic history of water on Mars and perform its extended science mission. MRO occasionally captured images of Phobos with its two instruments, High-Resolution Imaging Science Experiment (HiRise) and the hyperspectral infrared Compact Reconnaissance Imaging Spectrometer for Mars [6]. Those measurements and investigations have provided new insight into Phobos. However, many questions about this moon remain unanswered, including: What are the compositions and origins? What are the internal structures? What is the relationship of Mars' moons to each other? How did the striations/grooves form on Phobos, and in what sequence? Are the craters primary, secondary, or "sesquinary" [8]? These and other questions were mainly reported by Planetary Science Decadal Survey, [9], but they were also motivated by NASA's Journey to Mars, [10]. Engineers and scientists are considering using this moon in several different ways, such as staging areas for the trip to Mars, a teleoperation center for robots on Mars' surface to avoid long-latency, and as a place to build Mars descent heat shields by using silicates and other resources on Phobos [11]. Exploration of Phobos is not not driven by planetary science and the quest to understand the 
origins of this small body, but Phobos also holds strategic importance in developing a forward base for human exploration of Mars [12].

The Japan Aerospace Exploration Agency (JAXA) has been developing a mission dedicated to exploring Phobos called the Mars Moon eXploration (MMX) [13,14]. This sample return mission will help clarify the moon's origin, its composition, and understand the circum-Martian environment. The launch of MMX is planned for 2024, with five years of mission duration. MMX will be in a quasi-stationary orbit to perform mineralogy mapping, imaging for geological studies, close-up observations for landing-site selections, and internal structure investigations [13]. While MMX remains in its design phase, its science data will supersede that of any CubeSat mission. However, the failures of the three flagship-class missions to Phobos have cautioned us to consider a multi-pronged approach to exploration and not to put all our eggs in one basket. Depending on the success of MMX, Perseus can be re-tooled to attain complementary science objectives, targeted follow-on investigations of specified areas of interest, or re-engineered with a similar strategy to perform the first detailed study of the other small Martian moon, Deimos. CubeSats and small satellites offer a new low-cost option to perform interplanetary exploration.

JPL's INSPIRE is one such attempt that will result in a pair of CubeSats dropped off in an Earth escape trajectory [15]. INSPIRE is a pair of CubeSats that will fly past the moon to perform a technical demonstration. It includes a magnetometer, a deep space X-band communication system, a computer, and electronics. Another proposed interplanetary spacecraft is the Hummingbird jointly proposed by NASA Ames and Microcosm [16]. Hummingbird is a spacecraft architecture intended to tour asteroids. It includes slots to carry CubeSats that would be deployed upon rendezvous with a target of interest. Additionally, it includes a telescope to observe an asteroid target at a distance. Another interplanetary CubeSat is LunaH-Map, a 6U CubeSat selected for a NASA SLS EM1 mission. LunaH-Map is a science-focused mission using an experimental miniature Neutron Spectrometer to map speculated water ice deposits in the permanently shadowed craters of the Lunar South Pole [17]. The mission, as with Lunar Ice Cube, will use an experimental Iodine fueled ion-thruster. Lunar Ice Cube is a similar mission that will use an Infrared Spectrometer to look for water ice in the Lunar South Pole [18]. Lunar Flashlight is a third planned mission to explore the Lunar South Pole for ice deposit. It would use laser spectroscopy to identify the presence of water ice. NEAScout is a proposed SLS EM1 mission intended to explore Near-Earth asteroids.

Another mission called LUMIO will travel to the $L_{2}$ Lagrange point and monitor the moon for meteor impacts [19]. The spacecraft would use a combination of solar sails and green monopropellant thrusters to reach an asteroid target [20]. The spacecraft contains cameras that would provide up to $10 \mathrm{~cm} /$ pixel resolution of an asteroid surface. Other mission concepts include BIRDY, a $3 \mathrm{U}$ CubeSat concept to collect radiation data between Earth and Mars [21]. Another series of CubeSat mission concepts were to be deployed from the Europa Multi-Flyby spacecraft on its 40+ flybys around Europa. Mission concept ranged from performing detailed observation of Europa's atmosphere [22] to close-flybys of Europa to obtain detailed surface imagery and look for plumes [23]. In comparison, the proposed CubeSat mission is an incremental advancement over JPL's MarCo CubeSats. The mission concept is novel for enabling a science-focused mission out of a spacecraft architecture intended for a technology demonstration. The proposed CubeSat has a similar functional architecture to MarCo except for using a green monopropellant system based on a proven hydrazine fueled thruster. The mission would, much like several EM1 missions, achieve capture orbit around Mars instead of the Moon. The spacecraft will then do a series of co-orbit flybys to get higher resolution thermal and visible camera images of Phobos.

CubeSats offer the opportunity to perform low-cost science exploration, with rapid turn-around times between multiple high-profile missions. The high failure rate of past Phobos missions must be considered in those future missions. An alternative option is to have multiple small spacecraft that focus on simple tasks instead of a single, large spacecraft with multitasking functions. For those reasons, it is a reasonable option to utilize CubeSats as stepping-stones to exploring Phobos, uncovering its 
mysteries, and contributing towards long-term human exploration of Mars. Due to the involvement of integer parameters and nonlinear functions, mission concept design problems can be formulated as mixed-integer, nonlinear programming (MINLP) problems [24]. Evolutionary algorithms, such as the Genetic Algorithm [25], have provided robust solutions to MINLP optimization. Genetic Algorithms have also been applied to spacecraft communication systems [26] and trajectories [27]. Our previous research developed automated software tools to design reconnaissance missions to planetary moons [28-31] using evolutionary algorithms. In this work, we extend these tools to design the trajectories for the Perseus mission concept.

\section{Science}

By obtaining a uniform, high-resolution visible and thermal coverage of Phobos (Table 1), Perseus shall enable a global characterization of Phobos geology, which is not obtainable at present. This includes visible and thermal maps that shall be utilized by future Phobos missions and made available to scientists, mission planners, and the In-situ Resource Utilization (ISRU) community. Such a dataset shall reduce the risk of future robotic surface missions and shall be useful in analyzing the feasibility and site location of a possible future human base on Phobos. Perseus visible and thermal images shall reveal, more consistently and globally than before, how the grooves and craters of Phobos overprint each other (sequence of superposition) and how the grooves vary photometrically and how they correlate with thermal inertia that is representative of the particle size or matrix. Based on existing datasets, especially the very limited thermal data that exist to date, such a correlation is not currently possible.

Specifically, the thermal images of Perseus enable temperature measurements at different times of the day, to an accuracy of $50 \mathrm{mK}$, which will provide data on the extent of the diurnal temperature variations across the surface of Phobos to resolutions of $100 \mathrm{~m} /$ pix. High amplitudes increase the likelihood of thermal fatigue and fracture of rocks and boulders on the surface [32], which may contribute to the production of fine regolith. Thermal measurements taken by Perseus can also be used to estimate surface roughness [33], which can, in turn, be used to assess favorable locations for future investigation or landing sites. Rough topography can indicate the presence of cold traps in shadows at high latitudes where unbound volatiles can migrate [34,35]; for example, water molecules have been found in cool, shadowed regions on the surface of the Moon [36]. Although Phobos exhibits high variations in its obliquity [37], which can make its cold traps transient, these rough regions are promising places to look for volatile-rich material and are thus important to find and assess.

Phobos has also been discovered to exhibit two distinct layers of red and blue fine grain sand, characterized by their spectral properties [38]. These geological units are exhibited because of the surface grain properties and have been observed on the tidally-locked near side of Phobos to Mars. More observations are needed to see the evolution and changes of these units and their interaction with solar winds. These interactions result in natural excavation of highly sloped surface, movement of sand grains and overall changes in the Moon's surface topography. Perseus would provide the opportunity for multiple flybys lasting at least a few months and that would provide numerous view of Phobos, particularly the red and blue geological units, including periodic variation in dynamics slopes and overall surface grain motion. Short of landing on Phobos, these rich observations can provide insight into the surface environment of Phobos and potentially discover the rich, complex dynamics and constant changes occurring on the surface of Phobos.

In addition to the thermal imaging, Perseus will also be able to image Phobos' surface from multiple perspectives, including its striations/grooves at resolutions greater than $10 \mathrm{~m} /$ pix (striation width about $100 \mathrm{~m}$ ). These images, which will be taken at similar illumination, will provide topographic details (heights and depths) of these curious striation features to learn more about their formation and evolution. These images will also allow assessments of surface boulder and crater topography: with over $50 \%$ coverage at these resolutions, Perseus will provide refined estimates of boulder size-frequency distributions, which have important implications on the body's material properties [39] 
and its formation and bombardment history [40]. The science requirements of Perseus, as shown in Table 1, are simple but significant: to return (1) thermal and (2) visible images of more than half the surface of Phobos, at a resolution greater than what is currently available. Detail characterization of the surface of Phobos can provide greater insight into the origin of Phobos, particularly if it was formed from Martian debris or is a captured asteroid. The highest priority regions to be photographed are the Northern and Southern Poles that face continuous sunlight during summer and winter, respectively, as shown in Figure 1. The described mission goals shall be achieved with a science payload consisting of a visible and thermal imager. The obtained data would be used by planetary scientists to better model and understand the origin and evolution of Phobos. The data would be used by future mission planners to assess the opportunities and risks in a future surface mission to Phobos. This could include down-selection of candidate landing sites, identification of surface hazards, and detailed plans for surface exploration. The science instruments drive mission requirements for orbital distance and mission duration to produce appropriate coverage and resolution. To achieve the stated science goals, the spacecraft needs to have several important capabilities, as summarized in Section 5.
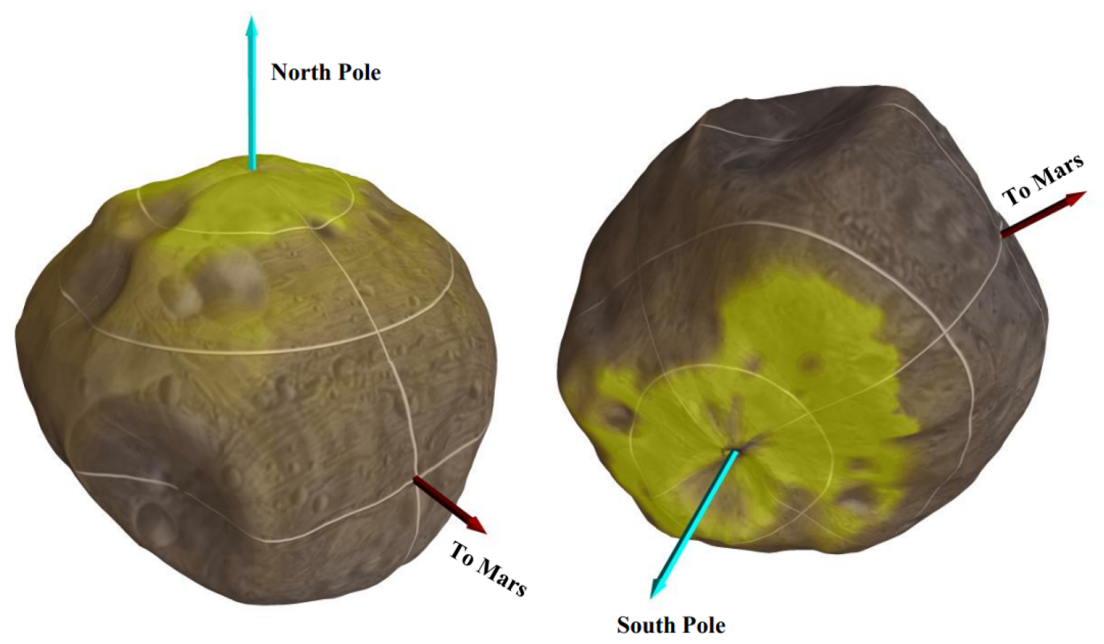

Figure 1. 3D Visualization of Phobos (Courtesy: Hopkins et. al. [41]). Regions in yellow face continuous sunlight and are the high priority targets for Phobos reconnaissance.

\section{Concept of Operations}

Figure 2 shows the Concept of Operations of the Perseus mission. Perseus is intended to be a secondary payload on a rocket heading on a Mars transfer orbit. Once the primary payload has reached a safe distance, the Canisterized Satellite Dispenser (CSD) will release Perseus, and it shall begin its deployment sequence. Immediately after ejection, the solar arrays and antennas shall passively unfold, allowing for solar power and communications capabilities. The satellite shall perform a series of systems checks followed by trajectory correction maneuvers and hibernation during its heliocentric cruise to Mars. 


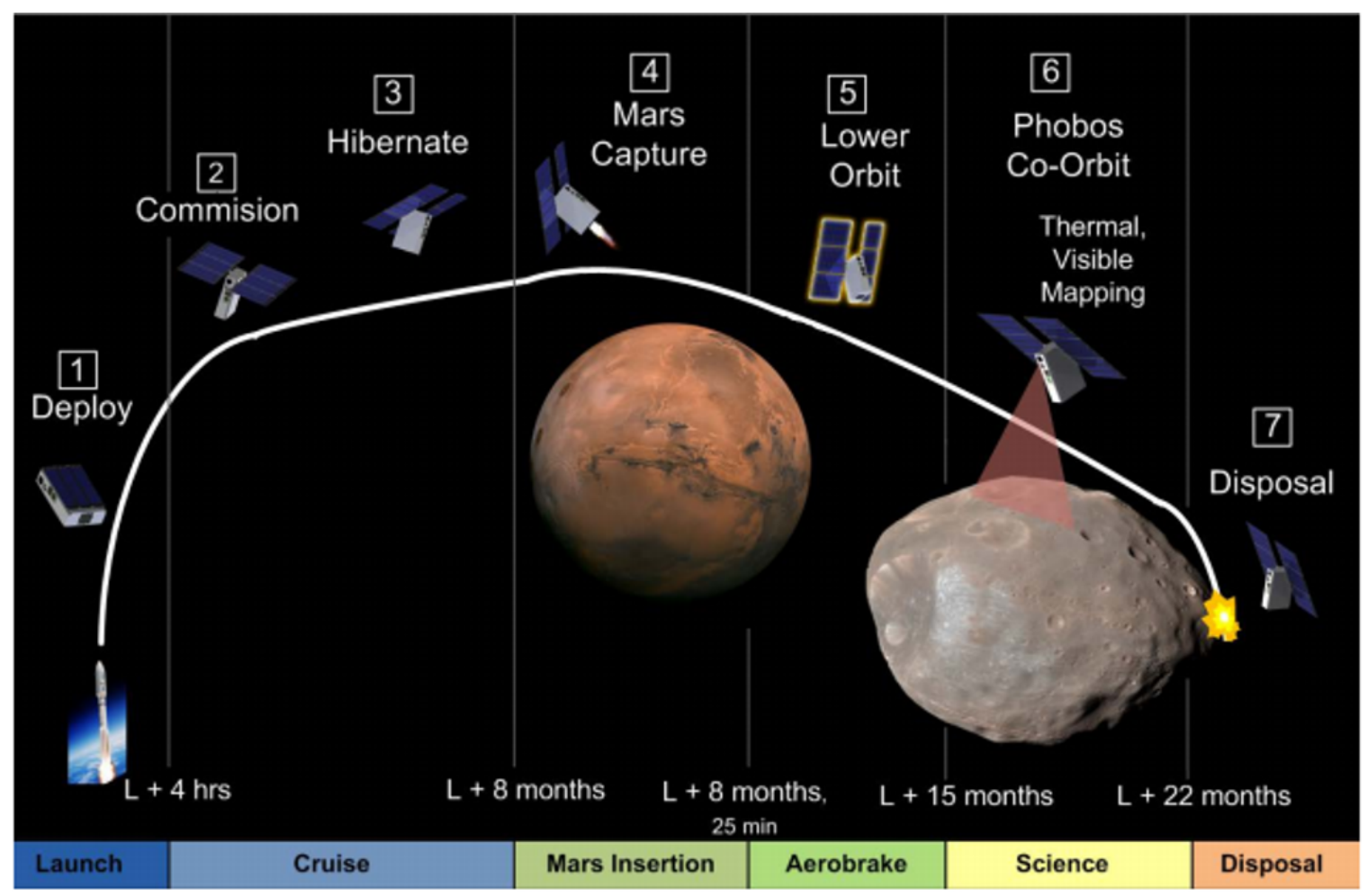

Figure 2. Concept of Operations of the Perseus mission to Phobos.

After launch, the launch vehicle's initial orbit insertion shall put the spacecraft on a hyperbolic trajectory with respect to Earth. The spacecraft will perform orbit correction maneuvers at different intervals during the heliocentric cruise to arrive at Mars. During this period, the satellite shall perform comprehensive system checks and refine its routine to prepare for the approaching science mission. The majority of the cruise duration shall be spent in a state of hibernation with the exception of thermal control systems, attitude control systems, and occasional communications. In the nominal mission concept, the spacecraft shall utilize aeroassist maneuvers and a series of strategic burns to lower the orbit and achieve a plane change to co-orbit with Phobos. Once the spacecraft is in a co-orbit, it shall frequently encounter Phobos and have opportunities to perform flyover imaging. This shall proceed until the end of the mission, at which point the option of impacting Perseus into Phobos shall be considered if it can be observed by Mars Express or other Mars orbiting assets. As an alternative, the spacecraft will directly flyby Phobos in a single hyperbolic pass to achieve its imaging objectives. In this case, the communications with the spacecraft would be terminated at its end of life, once its images are downlinked to the ground station on Earth.

\section{Spacecraft}

To take advantage of the small spacecraft technology, the Perseus spacecraft is populated with commercial off-the-shelf (COTS) hardware where applicable. It should be emphasized here that, since the Perseus mission is in a concept design phase, the hardware choices are preliminary and need further testing and analysis before final selection. The main reasons for selecting these components are attributed to their demonstrated legacy in spacecraft missions and our experience in working with them on other CubeSat missions [42,43]. Additionally, this also allows us to generate a preliminary mass budget of the baseline spacecraft required for maneuver planning. The baseline spacecraft of the Perseus mission uses a CubeSat with a 27U form factor, as shown in Figure 3. The individual spacecraft subsystems are described as follows. 


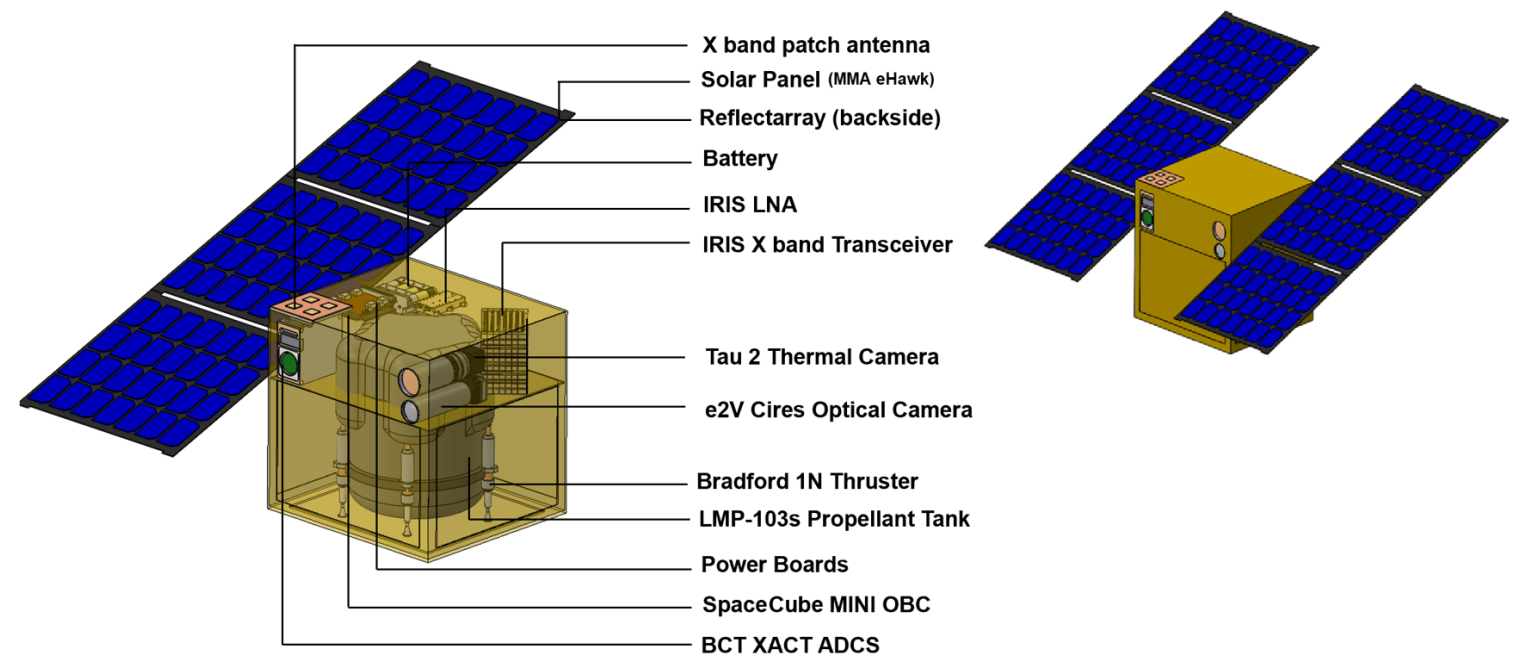

Figure 3. Spacecraft Layout with components shown (Left). Spacecraft fully assembled with pair of solar panels (Right).

\subsection{Science Payload}

To meet its science goals, Perseus will utilize a COTS assembled thermal and visible cameras, which are shown in Figure 4. The thermal camera is comprised of FLIR's Tau 2 with an NFOV lens and a Cameralink module. FLIR cameras are widely used in consumer thermal imaging, and Tau 2 is being used as a primary instrument in an upcoming US Air Force sponsored mission called THESIS [44]. The E2V camera was previously demonstrated on the repair of the Hubble space telescope [45] and the CheMin instrument onboard the Curiosity rover [46]. However, both instruments have not been flown in deep space, and hence the camera needs to be space-qualified.
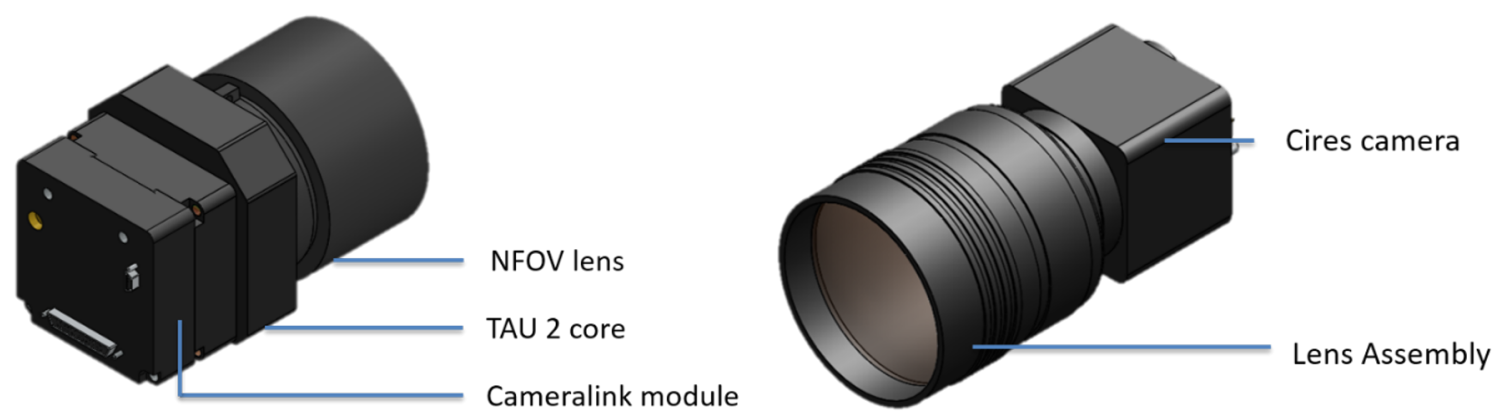

Figure 4. Thermal camera (left) and visible camera (right) assembly.

The science phase of the mission will begin once the spacecraft is located at an altitude $h_{T}$ below $100 \mathrm{~km}$ with respect to Phobos, as shown in Table 2. The baseline mission is designed such that the spacecraft spends much of its time in a feasible region defined by altitudes between 100-3 km over the surface of Phobos. This allows us to obtain $0.34 \mathrm{~m} /$ pix scale visible images and $1.5 \mathrm{~m} / \mathrm{pix}$ scale thermal images, which can be used for future geological studies and landing site determination for in-situ missions. 
Table 2. Spatial resolution comparison between the two payloads at various spacecraft working distances.

\begin{tabular}{|l|c|c|c|c|}
\hline Working Distance & $100 \mathrm{~km}$ & $50 \mathrm{~km}$ & $25 \mathrm{~km}$ & $3 \mathrm{~km}$ \\
\hline $\begin{array}{l}\text { Visible Camera } \\
\text { Spatial Resolution } \\
\text { (m/pix) }\end{array}$ & 10.59 & 5.29 & 2.12 & 0.34 \\
\hline $\begin{array}{l}\text { Thermal Camera } \\
\text { Spatial Resolution } \\
\text { (m/pix) }\end{array}$ & 49.5 & 24.75 & 12.37 & 1.5 \\
\hline
\end{tabular}

\subsection{Propulsion}

As a baseline system, we identified the Bradford ECAPS 1N LMP-103s thrusters for the spacecraft maneuvers. The propulsion system will use mono-propellants such as LMP-103s and AF-M 315E (specific impulse of about $240 \mathrm{~s}$ ) as they have a long shelf life and are of reduced toxicity, making it relatively simple to integrate with the rest of the CubeSat [47]. The thrusters will be mounted at the corners and center of the propulsion tanks, at a canted angle of $5^{\circ}$ for reaction wheel desaturation. While an exact fuel budget is not possible at an early concept development stage, we budget a propellant mass of $15 \mathrm{~kg}$ for all spacecraft maneuvers.

\subsection{Attitude Determination and Control System}

The Attitude Determination and Control System (ADCS) selected for Perseus underwent a series of design trade studies. Several integrated commercial ADCS solutions exist. Several of these integrated ADCS systems have been flown on CubeSats into Low Earth Orbit. Currently, only the BCT XACT system has flown in deep space on the MarCO mission [2]. This ADCS is involved in the successful operation of all subsystems and during all mission phases, such as the camera pointing, the solar panel pointing, and the reflector array pointing. For this reason, BCT XACT was selected as the main component for this system, and it is equipped with a star tracker, IMU, sun sensor, and three-axis reaction wheels. The thrusters described above are utilized for desaturating the reaction wheels.

\subsection{Command and Data Handling}

The Command and Data Handling $(\mathrm{C} \& D H)$ tasks are handled by the spacecraft On-Board Computer $(\mathrm{OBC})$. Since the $\mathrm{OBC}$ of Perseus will operate around Mars, we require that this system should sustain the Martian radiation environment [48]. Additionally, the selected OBC system should also be able to interface with other spacecraft subsystems. Based on this, the SpaceCube MINI was chosen, primarily for its high radiation tolerance. It was used in the IPEX mission [49] and has a legacy with its previous version, which flew on the Relative Navigation Sensors (RNS) experiment [50]. This OBC system consists of a primary Xilinx Virtex-5QV space-qualified processor and a daughter board with Aeroflex UT6325 FPGA, which acts as a watchdog, configuration manager, and scrubber. The primary board of the SpaceCube MINI provides 4 GB of Flash memory. The selected C\&DH architecture provides 8 Low Voltage Differentiating Signal (LVDS) interfaces and 8 RS-422 interfaces, which can satisfy interface requirements of several standard spacecraft hardware.

\subsection{Communications}

The communications system of Perseus will send science and telemetry data to Earth and additionally receive command signals from the ground station. We design the Perseus mission concept assuming the use of NASA's Deep Space Network (DSN) for communication and tracking [51]. Because of its demonstrated legacy on the MarCO mission [2], the Iris 2.1 module developed by the NASA Jet Propulsion Laboratory radio is used as the baseline transponder for the spacecraft. The Iris transponder uses the Xilinx Virtex 6 FPGA, which can be interfaced with the OBC module using the SPI interface. 


\subsection{Power System}

The electrical power system (EPS) uses a combination of electronics from the OBC integrated board and a set of auxiliary power boards to meet the interface requirements of the spacecraft subsystems. The spacecraft generates power using a pair of deployable solar panels from MMA Design. The form factor of the selected MMA E-HaWK solar panels varies from $2 \mathrm{U} \times 3 \mathrm{U}$ (stowed) to $6 \mathrm{U} \times 3 \mathrm{U}$ panel upon deployment. The spacecraft is baselined to use two GOMspace NanoPower BPX v3 Li-ion batteries as its power supply. The estimated mass and volume budgets, including a 15\% contingency for each subsystem, are presented in Table 3. As seen here, the nominal spacecraft is designed with a wet mass of $35.1 \mathrm{~kg}$, of which $20 \mathrm{~kg}$ is allotted for spacecraft maneuvers. Therefore, the dry mass of $20.1 \mathrm{~kg}$ is used to estimate the fuel requirements of the co-orbital mission concept.

Table 3. Mass and volume budgets of the Perseus mission.

\begin{tabular}{|c|c|c|c|c|c|c|c|c|}
\hline Subsystem & Component & Qty & $\begin{array}{c}\text { Mass } \\
\text { (kg) }\end{array}$ & Contingency (15\%) & Total Mass (kg) & $\begin{array}{c}\text { Vol. } \\
{\left[\mathrm{mm}^{3}\right]}\end{array}$ & Vol. Margin (15\%) & Total Vol. $\left[\mathrm{mm}^{3}\right]$ \\
\hline C\&DH & SpaceCube Mini & 1 & 0.3 & 0.045 & 0.35 & 170,000 & 25,500 & 195,500 \\
\hline $\mathrm{C} \& \mathrm{DH}$ & Mission Unique Card & 1 & 0.15 & 0.023 & 0.17 & 17,390 & 2609 & 19,999 \\
\hline Power & Battery & 2 & 0.365 & 0.055 & 0.84 & 347,020 & 52,053 & 798,146 \\
\hline Power & EPS & 1 & 0.043 & 0.006 & 0.05 & 27,056 & 4058 & 31,114 \\
\hline Power & Solar Panels & 2 & 0.98 & 0.147 & 2.25 & 171,677 & 25,752 & 394,857 \\
\hline Comms & IRIS X band Radio & 1 & 1 & 0.150 & 1.15 & 500,000 & 75,000 & 575,000 \\
\hline Comms & Patch Antenna & 1 & 0.05 & 0.008 & 0.06 & 54,616 & 8192 & 62,808 \\
\hline Comms & IRIS SSPA & 1 & 0.15 & 0.023 & 0.17 & 86,344 & 12,952 & 99,295 \\
\hline Comms & IRIS LNA & 1 & 0.08 & 0.012 & 0.09 & 44,021 & 6603 & 50,625 \\
\hline ADCS & BCT XACT & 1 & 0.91 & 0.137 & 1.05 & 467,016 & 70,052 & 537,068 \\
\hline Propulsion & $\begin{array}{c}\text { Bradford } 1 \text { N HPGP } \\
\text { Thruster }\end{array}$ & 5 & 0.38 & 0.057 & 1.09 & 34,091 & 5114 & 196,023 \\
\hline Propulsion & Propellant Tank & 1 & 1.8 & 0.270 & 2.07 & 571,107 & 85,666 & 656,773 \\
\hline Propulsion & Propellant & 1 & 15 & 2.250 & 21.30 & $16,840,607$ & $2,526,091$ & $19,366,699$ \\
\hline Structure & Chassis & 1 & 3.5 & 0.525 & 4.03 & $1,608,981$ & 241,347 & $1,850,328$ \\
\hline Instruments & Tau 2 Thermal cam \& lens & 1 & 0.21 & 0.032 & 0.24 & 77,342 & 11,601 & 88,943 \\
\hline Instruments & E2V Cires \& lens & 1 & 0.18 & 0.027 & 0.21 & 65,012 & 9752 & 74,764 \\
\hline Total & & & & & 35.11 & & & $24,997,943$ \\
\hline Margin $(27 \mathrm{U}, 54 \mathrm{~kg})$ & & & & & $35 \%$ & & & $36 \%$ \\
\hline
\end{tabular}

\section{Trajectory Design}

This section describes the trajectory design of the Perseus mission concept in order to satisfy the imaging requirements of its science objectives. For the purpose of mission concept design, we model the motion of spacecraft and Phobos assuming a spherical source of gravity from Mars [52]. Furthermore, the influence of spacecraft motion due to gravity from Phobos is not considered. As mentioned above, Perseus is intended to be a co-orbital exploration, where we explore Phobos by entering into Martian orbits. However, as a descoping scenario, we also examine an alternative mission concept where we explore Phobos through a hyperbolic flyby of Mars. While the reconnaissance methodology is different for these two concepts, we require that these alternate concepts share the same launch window. For this reason, we decouple the mission design into three different design problems:

- Heliocentric Cruise

- Co-orbital Mission Concept

- Hyperbolic Mission Concept

The heliocentric cruise problem will identify the Earth launch and Mars arrival epochs through Lambert arcs [52]. The co-orbital reconnaissance design will identify the feasible encounter resonance and nominal aerobraking parameters in addition to the encounter location. Finally, the hyperbolic reconnaissance design identifies the spacecraft's encounter location such that the relative velocities during the encounter are minimized. These problems are formulated as mixed-integer, nonlinear programming problems (MINLP), which are then solved using evolutionary algorithms such as the Genetic Algorithm [24]. The design of the mission concepts, in the current work, can better be described using a layered tree structure, as presented in Figure 5. It should be noted that, while the individual design problems in Figure 5 are solved using an end-to-end automated approach, the transition 
between individual problems is carried out manually. Specifically, the identified heliocentric cruise solution is passed as an input file to the hyperbolic and co-orbital mission concepts. We now explore the design space of individual problems. As described above, individual design optimization problems are solved using the Genetic Algorithm (GA) optimizer.

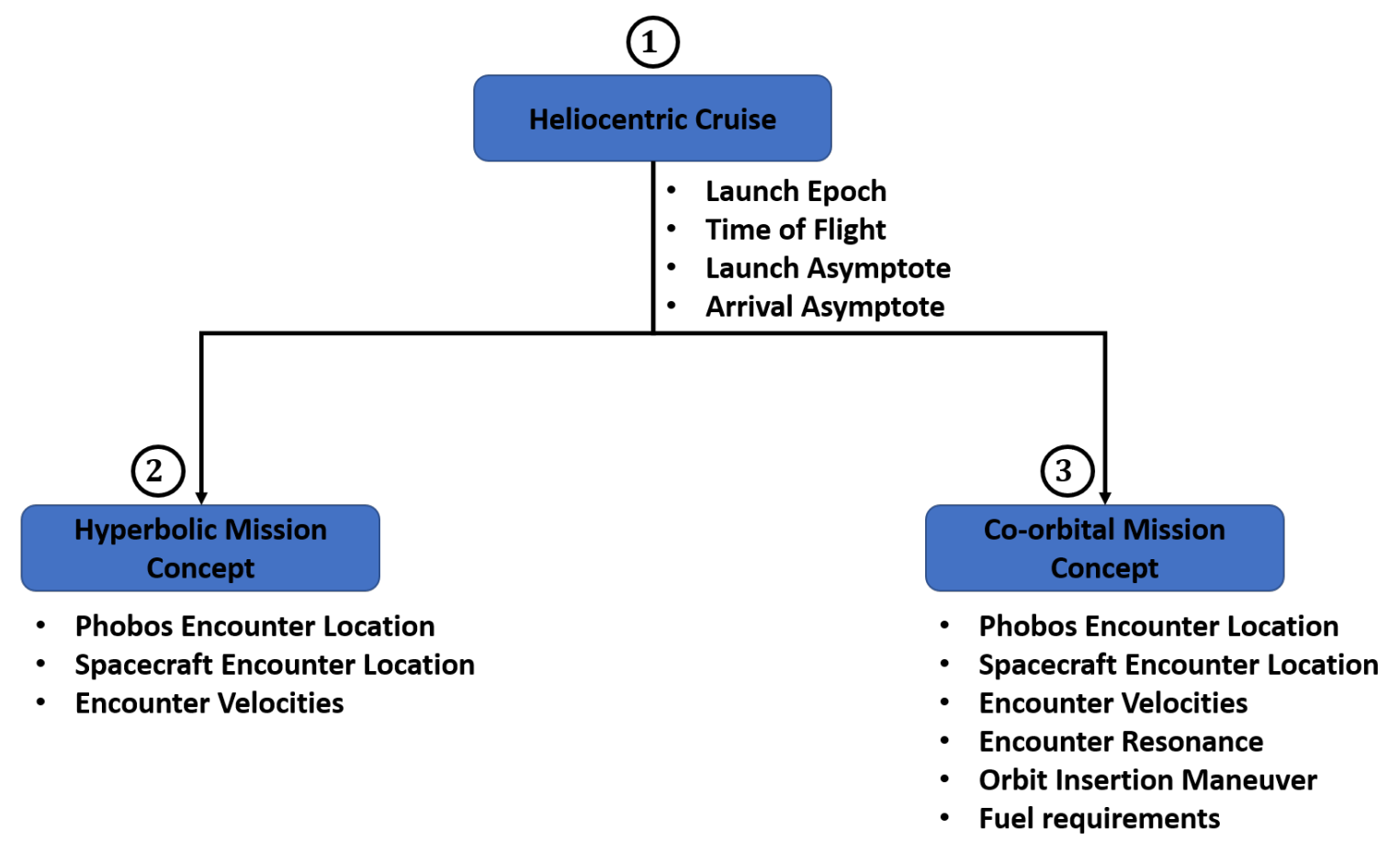

Figure 5. The layered tree architecture used in the current work to design the Perseus mission concept alternates.

\subsection{Heliocentric Cruise}

The heliocentric cruise design problem serves as the root node of all design problems, as seen in Figure 5. Here, we aim to identify the launch epoch $D_{L}$ at Earth and the time of flight ToF of the cruise to Mars. The arrival epoch $D_{A}$ can be computed from these parameters as

$$
D_{A}=D_{L}+T o F
$$

These epochs are used to solve Lambert's problem, where the terminal positions of the spacecraft and the time of flight are used to compute the spacecraft velocities at these terminal positions. The terminal velocities are used to determine the launch asymptote vector $\bar{V}_{\infty, 1}^{+}$at Earth, and arrival asymptote vector $\bar{V}_{\infty, 2}^{-}$. The asymptote vectors are crucial for mission concept design as they describe the relative velocity vectors and launch and arrival [52]. Specifically, the magnitude of launch asymptote can be used to compute the launch energy $C_{3,1}$ as:

$$
C_{3,1}=\left|\bar{V}_{\infty, 1}^{+}\right|^{2}
$$

The magnitude of arrival asymptote vector describes the relative velocity of the spacecraft with respect to Mars upon arrival at its sphere of influence [52]. We focus on the magnitude of the arrival asymptote $v_{\infty, 2}$ during the heliocentric cruise problem. However, we use the three-dimensional vector when designing the hyperbolic and co-orbital mission concepts. To simplify the calculations, 
we assume that the arrival asymptote is resolved in the Inertial $J_{2000}$ reference frame of Mars [52]. We can now formulate the hyperbolic cruise design as the following optimization problem:

$$
\begin{array}{cl}
\min & J_{H C}=w_{1}\left(\frac{C_{3,1}}{C_{3, \max }}\right)+w_{2}\left(\frac{v_{\infty, 2}}{v_{\infty, \text { max }}}\right)^{2} \\
\text { s.t. } & C_{3,1}-C_{3, \max } \leq 0 \\
& v_{\infty, 2}-v_{\infty, \max } \leq 0
\end{array}
$$

The parameters $w_{1}, w_{2}$ denote weights describing the importance of minimizing $C_{3,1}$ and $v_{\infty, 2}$. In addition to the constraints on $C_{3,1}$ and $v_{\infty, 2}$ in Equation (3), the design variables are assumed to bounded. In this work we refer to the vector containing the design variables as the design gene of the optimization problem. Figure 6 presents a map of the heliocentric cruise design gene.

\begin{tabular}{|c|c|c|}
\hline Parameter & Launch epoch & Time of Flight \\
\hline Variable & $D_{L}$ & ToF \\
\hline Range & $\begin{array}{c}\text { Integer } \\
{\left[D_{L, \min }, D_{L, \max }\right]}\end{array}$ & $\begin{array}{c}\text { Integer } \\
{\left[T o F_{\min }, T o F_{\max }\right]}\end{array}$ \\
\hline
\end{tabular}

Figure 6. The gene map of the heliocentric cruise design problem describing the different design variables and their bounds.

The launch epoch is enumerated using the Julian time format. Furthermore, the ephemerides of Earth and Mars, which are required to compute the $\bar{V}_{\infty, 1}^{+}$and $\bar{V}_{\infty, 2}^{-}$asymptotes, are modeled using an analytical model dependant on the Julian time [52]. It can be noted from Figure 6 that the design variables are modeled as integers. This is done in order to determine the launch window with a coarse resolution of one day. The parametric bounds in Equation (3) and Figure 6 are supplied to the optimizer at the beginning of the automated heliocentric cruise design.

\subsection{Co-Orbital Mission Concept}

As the nominal mission concept, the Perseus spacecraft will explore Phobos on Martian Orbits as illustrated in Figure 7. These co-orbits will be designed in such a way that the encounters repeat after $p$ orbits of Phobos or $q$ orbits of the spacecraft. The integers $p$ and $q$ are referred to as the resonance indices of the co-orbit [28]. The resonant co-orbit will be shaped such that it has a periapsis altitude of $h_{p, C}$ and allows the spacecraft to encounter the moon at a specified true anomaly $f_{v}$ as shown in Figure 7.

The encounter position of spacecraft relative to Phobos $\bar{R}_{f}$ is parameterized by spherical co-ordinates: flyby altitude $h_{f}$, right ascension $\theta_{x}$, and the declination angles $\theta_{y}$, as shown in Figure 8 . It is noted here that $h_{f}$ is not necessarily the closest flyby altitude, but an altitude which satisfies the minimum imaging requirements of the mission $\left(h_{T}<3 \mathrm{~km}\right)$. While the parameters $p, q, f_{v}, r_{f}$, $\theta_{x}$, and $\theta_{y}$ are sufficient to describe a resonant co-orbit [29], the maneuver cost associated with orbit insertion should also be factored into to the design. As described above, the Perseus spacecraft will use an aeroassist from Mars to be captured into its targeted resonant orbit. For the purpose of mission concept design, the aerobraking can be assumed to occur in four stages [52]:

- HEO capture

- Walk-in

- Main phase

- Walk-out 


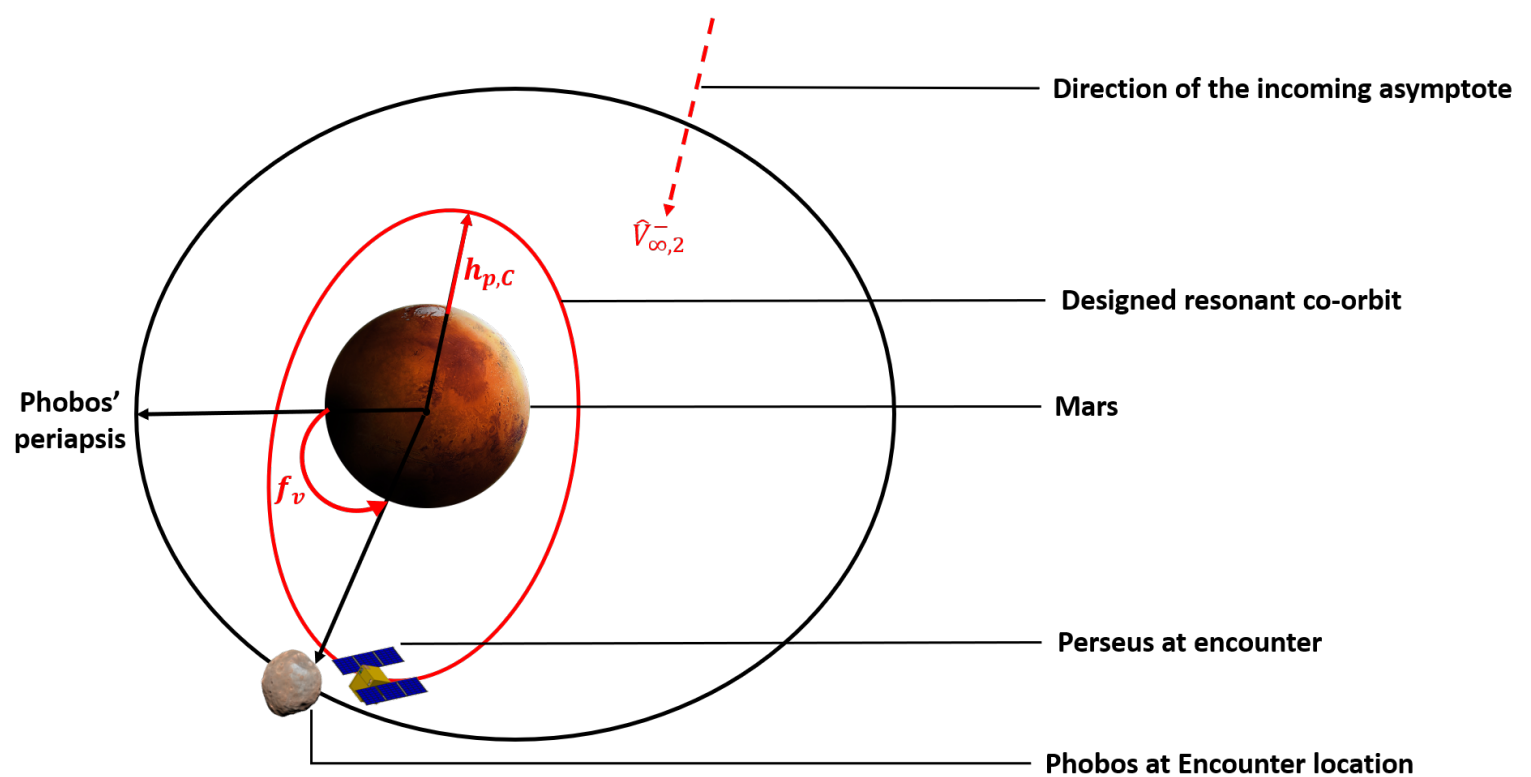

Figure 7. Illustration of the co-orbital mission concept highlighting the orbital parameters involved in designing the resonant co-orbit.

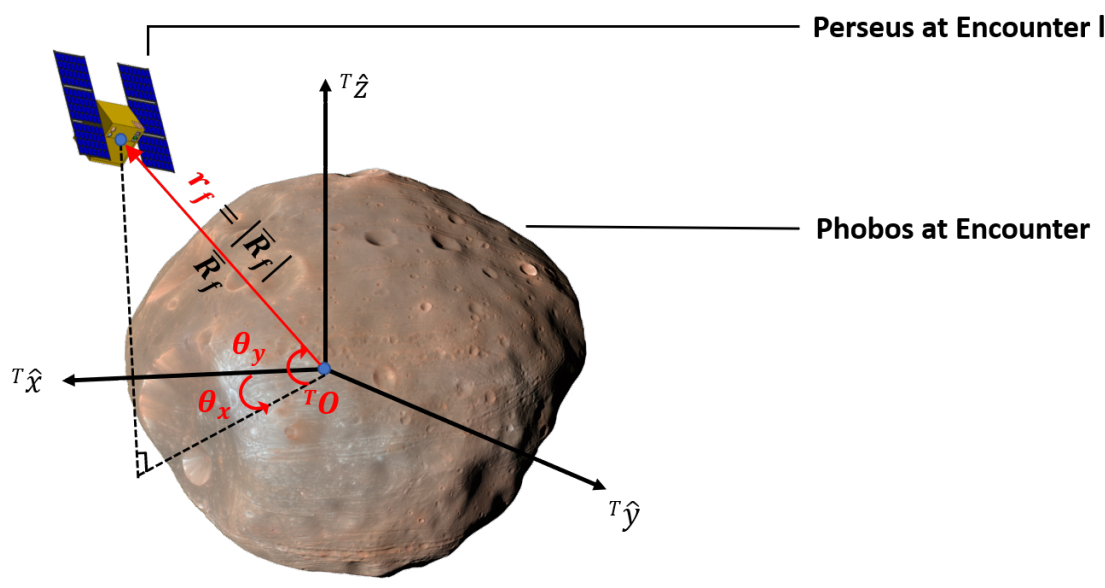

Figure 8. Illustration of the geometrical parameters required to define the spacecraft location with respect to Phobos during an encounter.

The HEO capture phase refers to the trajectory segment which arrives at the central planet (Mars) on the $\bar{V}_{\infty, 2}^{-}$asymptote and performs a tangential maneuver on its planetary periapsis at an altitude $h_{p, 0}$. This maneuver allows the spacecraft to enter into a high eccentric orbit (HEO) of eccentricity $e_{0}$. In the Walk-in phase, the spacecraft performs a tangential maneuver at its apoapsis to reduce its periapsis at an altitude $h_{A B}$, which subjects the spacecraft to significant atmospheric drag. This begins the Main phase of the aerobraking maneuver, where the spacecraft lowers its apoapsis altitude upon passing through the atmosphere. Finally, once the spacecraft reaches the apoapsis altitude of the specified resonant co-orbit, the spacecraft will begin its Walk-out phase, where it performs a tangential maneuver at its apoapsis to raise its periapsis altitude and enter into the resonant co-orbit. In this model, the spacecraft is assumed to expend fuel during the HEO capture, Walk-in, and Walk-out phases using in-plane, tangential maneuvers. Let $\Delta v_{O I}$ denote the net orbit insertion maneuver resulting from these three planar maneuvers. During the main phase, the spacecraft is not assumed to expend any fuel. While not strictly true [53], this assumption allows us to use analytical relationships to estimate the 
spacecraft's fuel requirements. In addition to these in-plane maneuvers, the spacecraft will perform an orientation correction maneuver $\Delta v_{O C}$ to correct the orientation orbital elements, namely inclination, RAAN, and argument of periapsis, to be the same as the targeted resonant orbit. Therefore, the net maneuver cost associated with a specified resonant co orbit $\Delta v_{\text {net }}$ is given by

$$
\Delta v_{\text {net }}=\Delta v_{O I}+\Delta v_{O C}
$$

\section{Co-Orbit Design}

The gene map describing the design of the resonant co-orbit is presented in Figure 9. The algorithms to compute the orbital elements of the resonant co-orbits from the parameters in Figure 9 are presented in $[29,30]$ along with models to compute $\Delta v_{\text {net }}$. We desire that the selected co-orbit has a bounded maneuver cost $\Delta v_{\text {net }}$ associated with Martian capture, and a bounded periapsis altitude $h_{p, C}$.

\begin{tabular}{|c|c|c|c|c|c|c|c|c|}
\hline \multirow{2}{*}{ Parameter } & \multirow{2}{*}{$\begin{array}{c}\text { Hyperbolic } \\
\text { periapsis } \\
\text { altitude }\end{array}$} & \multirow{2}{*}{$\begin{array}{c}\text { HEO } \\
\text { eccentricity }\end{array}$} & \multicolumn{2}{|c|}{ Resonance parameters } & \multirow{2}{*}{$\begin{array}{c}\text { Altitude } \\
\text { of } \\
\text { encounter }\end{array}$} & \multirow{2}{*}{$\begin{array}{c}\text { Right ascension } \\
\text { of } \\
\text { encounter }\end{array}$} & \multirow{2}{*}{$\begin{array}{l}\text { Declination } \\
\text { of } \\
\text { encounter }\end{array}$} & \multirow{2}{*}{$\begin{array}{c}\text { Phobos' } \\
\text { True } \\
\text { anomaly }\end{array}$} \\
\hline & & & $\begin{array}{c}\text { \# Target } \\
\text { orbits }\end{array}$ & $\begin{array}{l}\text { \# spacecraft } \\
\text { orbits }\end{array}$ & & & & \\
\hline Variable & $h_{p, 0}$ & $e_{0}$ & $p$ & $q$ & $h_{f}$ & $\theta_{x}$ & $\theta_{y}$ & $f_{v}$ \\
\hline
\end{tabular}

Figure 9. The gene map of the resonant co-orbit design problem describing the different design variables and their bounds.

As noted in Table 2, the Perseus spacecraft's imaging duties begin when the spacecraft is below an altitude of $100 \mathrm{~km}$ from the surface of Phobos. Therefore, to maximize our science returns, it is desired that the spacecraft has a slow relative velocity with respect to Phobos and also spends a large amount of time in this encounter region. This decision allows us to maximize photon collection by the cameras and prevent defects such as motion blurs. Therefore, on a propagated co-orbit, we isolate the trajectory segment where $h_{T} \leq 100 \mathrm{~km}$ as the encounter segment Enc. As noted in Table 2, the minimum altitude of the spacecraft with respect to Phobos $\min \left(h_{T}\right)$ should be below $3 \mathrm{~km}$ to generate images of $0.34 \mathrm{~m} / \mathrm{px}$ (visual) or $1.5 \mathrm{~m} / \mathrm{px}$ (thermal) resolution. However, to avoid a spacecraft collision with Phobos, we place a constraint that $h_{f}>0$. The maximum radius of Phobos is used as a baseline to calculate the spacecraft altitude with respect to Phobos. Therefore, we can formulate the co-orbit design problem as

$$
\begin{array}{cl}
\min & J_{O r b}=w_{c, 1} \frac{\sum_{k \in E n c}\left|\bar{V}_{f, k}\right|}{T_{E n c}}+w_{c, 2} \Delta v_{O I}^{2}+w_{c, 3} \Delta v_{O C}^{2} \\
\text { s.t. } & \Delta v_{O I}-\Delta v_{O I, \max } \leq 0 \\
& \Delta v_{O C}-\Delta v_{O C, \max } \leq 0 \\
& h_{p, \min }-h_{p, C} \leq 0 \\
& \min \left(h_{T}\right)-h_{\text {Thr }} \leq 0 \\
& \text { Phobos collision }=\text { False }
\end{array}
$$

where $\left|\bar{V}_{f, k}\right|$ denotes the relative velocity of the spacecraft at time step $k$ and $T_{E n c}$ denotes the total time that the spacecraft spends inside the encounter region. The numerator in defining the objective function in $J_{O r b}$ in Equation (5) is selected to penalize the relative velocities at each time step during an encounter. Additionally, the denominator in $J_{O r b}$ is selected to maximize the encounter duration of the spacecraft with respect to Phobos. The weights $w_{c, 1}, w_{c, 2}$, and $w_{c, 3}$ are used to specify the relative importance of individual terms and to non-dimensionalize the objective function. The parametric bounds in Figure 9 and Equation (5) are supplied to the optimizer at the beginning of the automated co-orbit design. 


\subsection{Hyperbolic Mission Concept}

As described above, the hyperbolic mission concept is an alternative to the co-orbital mission in the case of descoping. The heliocentric cruise design problem described above provides the incoming asymptote $\bar{V}_{\infty, 2}^{-}$at Mars. The challenge now is to construct a hyperbolic trajectory that contains this incoming asymptote while encountering Phobos at a desired location, as shown in Figure 10.

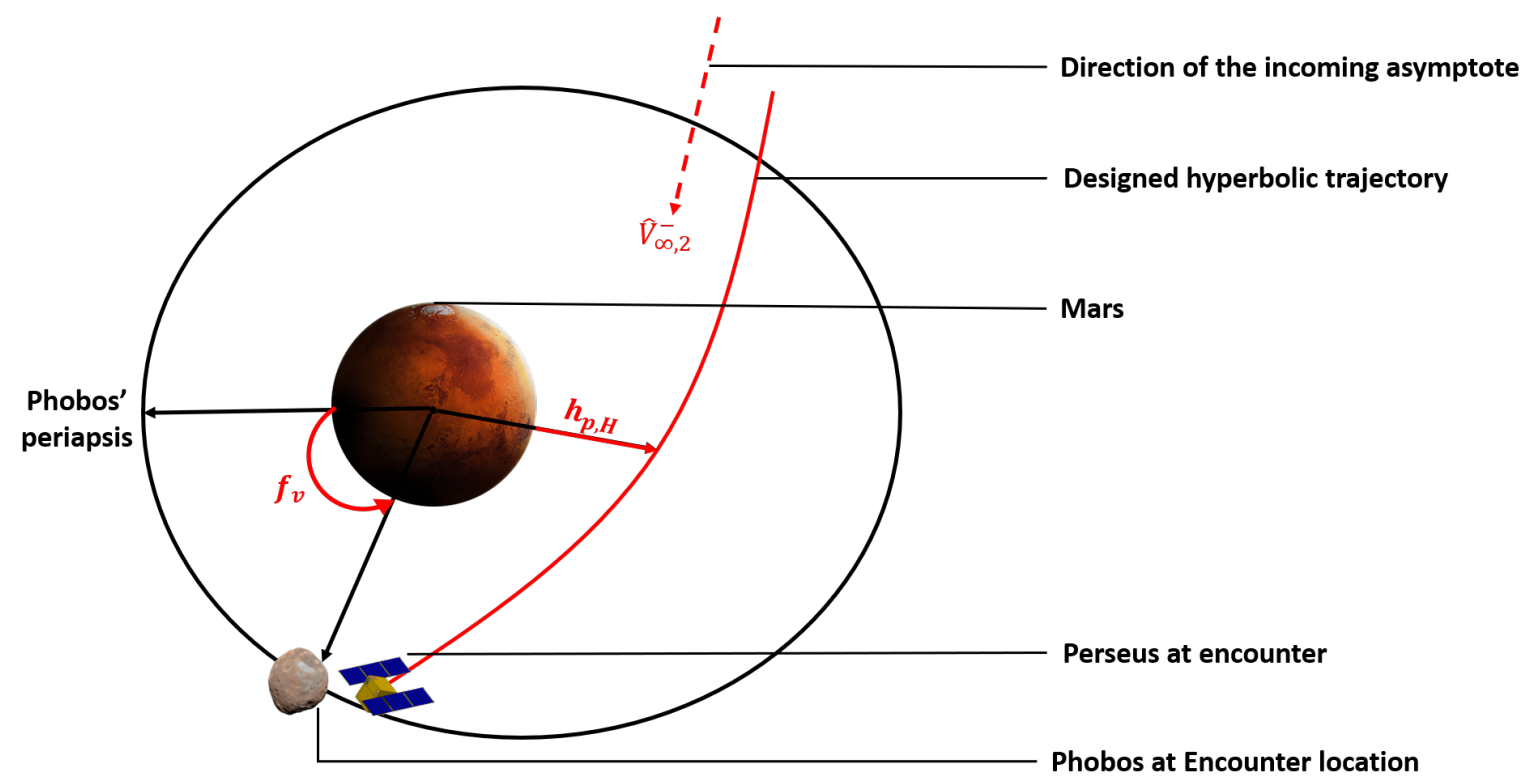

Figure 10. Illustration of the hyperbolic mission concept highlighting the parameters involved in designing the encounter hyperbolic trajectory.

\subsubsection{Hyperbolic Trajectory Construction}

We now derive the orbital elements of the hyperbolic trajectory which encounters a planetary moon at a desired location while ensuring that the spacecraft arrives on a specified hyperbolic asymptote. To derive these elements, we follow the same procedure described in Nallapu et. al. [31]. The encounter location of the moon is assumed to be parameterized by its true anomaly $f_{v}$. The encounter position of spacecraft relative to Phobos $\bar{R}_{f}$ is parameterized by spherical co-ordinates: flyby radius $r_{f}$, right ascension $\theta_{x}$, and the declination angles $\theta_{y}$ (Figure 8). It is noted here that $r_{f}$ is not necessarily the closest flyby altitude but a distance that satisfies the mission's imaging requirements. During an encounter, when Phobos is located at $\bar{R}_{T, v}$ corresponding to the true anomaly $f_{v}$, the position vector of spacecraft with respect to the planet, during the encounter, $\bar{R}_{P, v}$ can be found as

$$
\bar{R}_{P, v}=\bar{R}_{f}+\bar{R}_{T, v}
$$

Equation (6) specifies the spacecraft's inertial location during the encounter, while the incoming $\bar{V}_{\infty, 2}^{-}$is noted at an epoch when the spacecraft is at Mars' SoI. Therefore, to determine the complete spacecraft state at an encounter, a boundary value problem is formulated, which allows us to compute the velocity vector $\bar{V}_{i}$ with respect to the planet at the encounter epoch. Let $\bar{V}_{P, v}=\left[\begin{array}{lll}v_{x} & v_{y} & v_{z}\end{array}\right]^{T}$ be this unknown velocity vector. The Vis-Viva equation [52] allows us to constrain this velocity vector as

$$
v_{P}^{2} *=v_{x}^{2}+v_{y}^{2}+v_{z}^{2}=\left(v_{\infty, 2}^{-}\right)^{2}+\left(\frac{2 \mu_{M}}{r_{f}}\right)=v_{P}^{2}
$$


where $\mu_{M}$ is the gravitational parameter of Mars. The right superscript $*$ indicates that the term is a function of the unknown velocity components. The semi-major axis $a_{P}$ and eccentricity vector $\bar{e}_{P}$ of the hyperbola can be written as [52]

$$
a_{P}=-\frac{\mu_{M}}{v_{\infty, 2}^{2}}
$$

and

$$
\bar{e}_{P}=\frac{1}{\mu_{M}}\left(\left(v_{P}^{2}-\left(\frac{\mu_{M}}{r_{f}}\right)\right) \bar{R}_{P, v}-\left(\bar{R}_{P, v} \cdot \bar{V}_{P, v}\right) \bar{V}_{P, v}\right)
$$

The specific angular momentum $\bar{h}_{P}$ and orbit normal vector $\hat{n}_{P}$ can be expressed as

$$
\bar{h}_{P}=\bar{R}_{P, v} \times \bar{V}_{P, v}
$$

and

$$
\hat{n}_{P}=\hat{k} \times \frac{\bar{h}_{P}}{\left|\bar{h}_{P}\right|}=\hat{k} \times \hat{h}_{P}
$$

where $\hat{k}=\left[\begin{array}{lll}0 & 0 & 1\end{array}\right]^{T}$ is the $z$ axis of the central Mars $J 2000$ frame. The vectors expressed in Equations (9)-(11) are functions of the unknown velocity components. These can be used to define the orientation elements of the orbit. The right ascension of the ascending node $\Omega_{P}$, inclination $i n_{P}$, and argument of periapsis $\omega_{P}$ of the spacecraft can be determined as [52]

$$
\begin{gathered}
\Omega_{P}= \begin{cases}\arccos \left(\frac{\hat{n}_{P}[1]}{\left|\hat{n}_{P}\right|}\right) & \hat{n}_{P}[1] \geq 0 \\
2 \pi-\arccos \left(\frac{\hat{n}_{P}[1]}{\left|\hat{n}_{P}\right|}\right) & \hat{n}_{P}[1]<0\end{cases} \\
i n_{P}=\arccos \left(\frac{\hat{h}_{P}[3]}{\left|\hat{h}_{P}\right|}\right)
\end{gathered}
$$

and

$$
\omega_{P}= \begin{cases}\arccos \left(\hat{n}_{P} . \hat{e}_{P}\right) & \hat{e}_{P}[3] \geq 0 \\ 2 \pi-\arccos \left(\hat{n}_{P} . \hat{e}_{P}\right) & \hat{e}_{P}[3]<0\end{cases}
$$

Finally, the hyperbolic true anomaly $f_{\infty, i}$ is computed as

$$
f_{\infty}=\arccos \left(\frac{-1}{e_{P}}\right)
$$

where $e_{P}$ is the magnitude of $\bar{e}_{P}$ and the numbers inside [] indicate the components of the corresponding vector. These parameters can be used to determine the periapsis altitude $h_{p}$ as

$$
h_{p, H}=a_{p}\left(1-e_{P}\right)-R_{M}
$$

where $R_{M}$ is the radius of Mars. On the incoming asymptote, we set the true anomaly of the as $f_{i}=-f_{\infty}$. The asymptotic excess velocity vector can be expressed as

$$
\bar{V}_{\infty, 2}^{*}=R_{3}\left(-\Omega_{P}\right) R_{1}\left(-i n_{P}\right) R_{3}\left(-\omega_{P}\right)^{P} \bar{V}_{\infty, 2}^{-}
$$


where $R_{1}$ and $R_{3}$ are principal rotation matrices about Axes 1 and 3, respectively [54], and ${ }^{P} \bar{V}_{\infty, 2}^{-}$is the incoming excess velocity vector resolved in the perifocal frame [52] given by

$$
{ }^{P} \bar{V}_{\infty, 2}^{-}=\sqrt{\frac{\mu_{M}}{a_{P}\left(1-e_{P}^{2}\right)}}\left[\begin{array}{c}
\sin \left(f_{\infty, i}\right) \\
e_{P}+\cos \left(f_{\infty, i}\right) \\
0
\end{array}\right]
$$

The right ascension of arrival $R A A$ and declination of arrival $D A A$ of the spacecraft can now be extracted from the asymptote as

$$
R A A=\arctan \left(\frac{\bar{V}_{\infty, 2}[2]}{\bar{V}_{\infty, 2}[1]}\right)
$$

and

$$
D A A=\arcsin \left(\frac{\bar{V}_{\infty, 2}[3]}{\left|\bar{V}_{\infty, i}\right|}\right)
$$

This allows us to place final constraints on the trajectories as

$$
R A A^{*}=R A A
$$

and

$$
D A A^{*}=D A A
$$

The angles on the left-hand side of Equations (21) and (22) are functions of the unknown velocity components, while the right-hand side is computed from the incoming asymptote $\bar{V}_{\infty, 2}^{-}$noted from the hyperbolic cruise design problem. Equations (7), (21), and (22) are nonlinear functions of the spacecraft velocity components, and are solved using a nonlinear root solver. Specifically, the trust-region dogleg solver of MATLAB was used to compute the spacecraft velocity vector [55]. Through experimentation, the velocity of Phobos during the encounter was found to produce faster convergences to the solutions if used as an initial guess. The root solver error $\bar{\epsilon}$ can be defined as the three-dimensional vector

$$
\bar{\epsilon}=\left[\begin{array}{c}
v_{P}^{2}-v_{P}^{2} * \\
R A A-R A A^{*} \\
D A A-D A A^{*}
\end{array}\right]
$$

The magnitude of $\bar{\epsilon}$ is constrained during the trajectory design problem. The solutions to these equations allow us to compute the spacecraft's position and velocity vectors on the hyperbola during the Phobos encounter, thus specifying the complete hyperbolic trajectory.

\subsubsection{Hyperbolic Trajectory Design}

We can now formulate the hyperbolic trajectory design problem similar to Equation (5) as

$$
\begin{array}{ll}
\min & J_{H y p}=w_{H} \frac{\sum_{k \in E n c}\left|\bar{V}_{f, k}\right|}{T_{E n c}} \\
\text { s.t. } & h_{p, \min }-h_{p, H} \leq 0 \\
& \min \left(h_{T}\right)-h_{T h r} \leq 0 \\
& |\bar{\epsilon}|-\epsilon_{\max } \leq 0 \\
& \text { Phobos collision }=\text { False }
\end{array}
$$


where $w_{H}$ is a constant weight used to non-dimensionalize the objective function. The bounds in Equation (24) and Figure 11 are supplied to the optimizer at the beginning of the automated hyperbolic trajectory design.

\begin{tabular}{|c|c|c|c|c|}
\hline Parameter & $\begin{array}{c}\text { Radius } \\
\text { of } \\
\text { encounter point }\end{array}$ & $\begin{array}{c}\text { Right ascension } \\
\text { of } \\
\text { encounter point }\end{array}$ & $\begin{array}{c}\text { Declination } \\
\text { of } \\
\text { encounter point }\end{array}$ & $\begin{array}{c}\text { Phobos' } \\
\text { True anomaly }\end{array}$ \\
\hline Variable & $h_{f}$ & $\theta_{x}$ & $\theta_{y}$ & $f_{v}$ \\
\hline Range & $\begin{array}{c}\text { Real } \\
{\left[h_{\min }, h_{\max }\right]}\end{array}$ & $\begin{array}{c}\text { Real } \\
{[0,360]}\end{array}$ & $\begin{array}{c}\text { Real } \\
{[-90,90]}\end{array}$ & $\begin{array}{c}\text { Real } \\
{[0,360]}\end{array}$ \\
\hline
\end{tabular}

Figure 11. The gene map of the hyperbolic trajectory design problem describing the different design variables and their bounds.

\section{Results}

In this section, we explore the results of automated design problems described in Figure 5. We begin by exploring the design of heliocentric cruise. The incoming asymptote at Mars is then used in designing the nominal co-orbital mission and the descoped hyperbolic mission. All simulations were run on a high-performance computer cluster with a $1.8 \mathrm{GHz}$ Intel i7 Processor. Each design problem was solved five times to verify if the final selected solution was a local optimal solution. A stall limit of 100 iterations was used to select the final solution in each trial. The solutions of the individual mission designs are described as follows:

\subsection{Heliocentric Cruise}

The input parameters to the heliocentric cruise design problem are presented in Table 4. The launch epochs presented in Table 4, were enumerated in the Julian date format [52]. The weights were selected to emphasize the minimization of $v_{\infty, 2}$ since this influences the magnitude of the orbit insertion $\Delta v$. The ivLam solver, a fast Lambert problem solver, developed by the University of Texas [56], was used to design the heliocentric cruise from a given design gene.

Table 4. Parameters and bounds supplied as inputs to the heliocentric cruise design problem.

\begin{tabular}{|c|c|}
\hline Parameter & Value \\
\hline$\left[D_{L, \text { min }}, D_{L, \text { max }}\right]$ & [1 January 2022,31 December 2023] \\
\hline$\left[T o F_{\text {min }}\right.$, To $\left.F_{\text {max }}\right]$ & {$[1,400]$ days } \\
\hline$w_{1}, w_{2}$ & $0.2,0.8$ \\
\hline$C_{3, \max }$ & $50 \mathrm{~km}^{2} / \mathrm{s}^{2}$ \\
\hline$v_{\infty, \max }$ & $3 \mathrm{~km} / \mathrm{s}$ \\
\hline
\end{tabular}

The integer programming problem in Equation (3) was solved using the proprietary GA optimizer of MATLAB [25]. The optimizer used a tournament selection algorithm to select non-elite genes, along with special methods for crossover and mutation when handling nonlinear and integer programming problems $[57,58]$. The results of five GA optimization trials of the heliocentric cruise optimization are presented in Figure 12. Each generation of a trial evaluated 100 trajectory design genes. The subsequent search generations were populated with $20 \%$ elite genes, while $80 \%$ of the remaining genes were achieved through the crossover operation. A uniform stochastic selection criterion was used to select the parents for crossover and mutation operations. The minimum fitness of all GA trials was found to be 0.599 . 

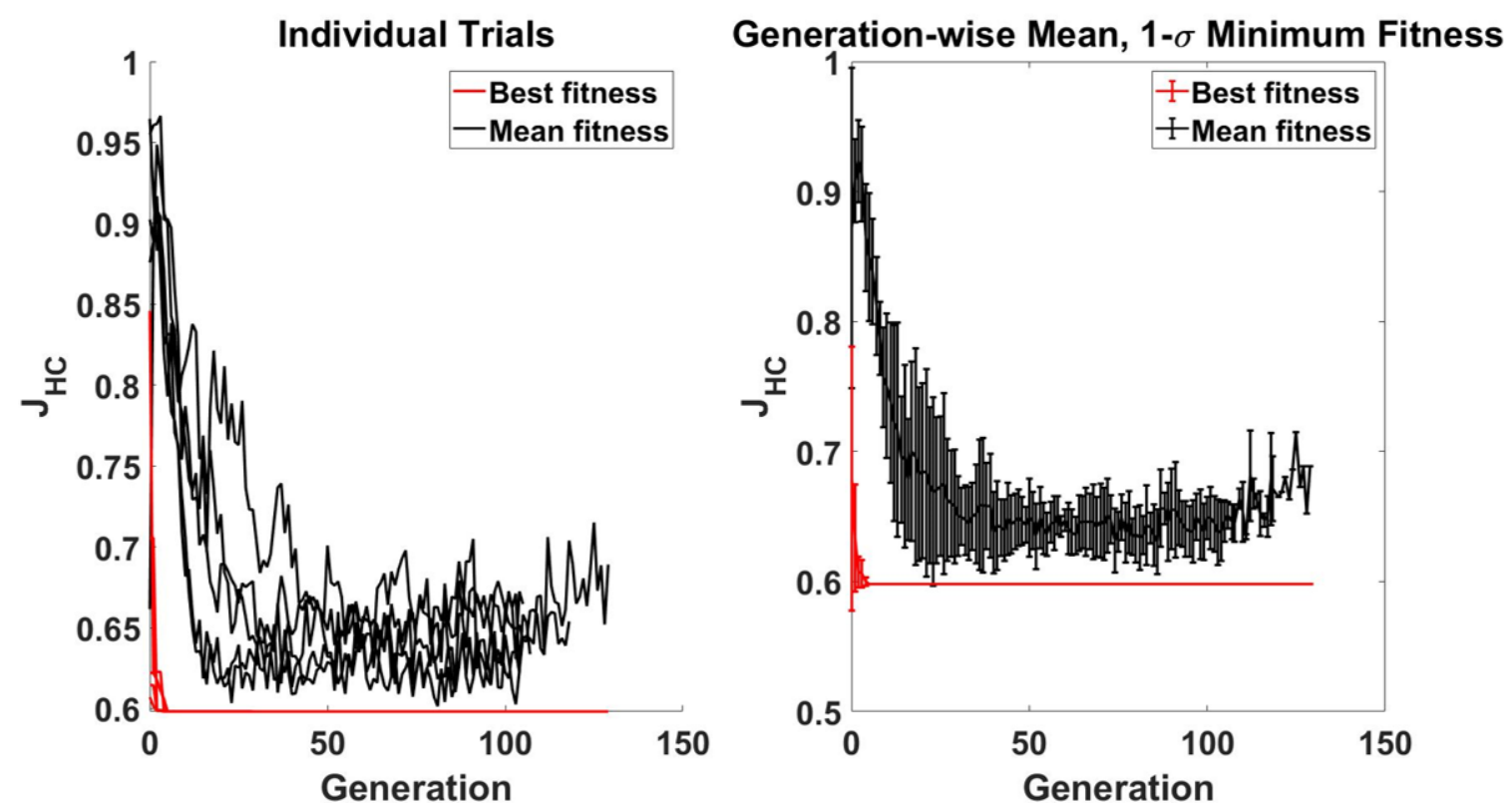

Figure 12. Variation of the heliocentric cruise fitness for the Perseus mission concept across different GA populations generations showing: the individual optimization trials (left); and their statistical distribution (right).

The final generations of all five trials identified 424 feasible heliocentric cruise design solutions whose figures of merit are presented in Figure 13 along with the Earth-Mars porkchop plot [52]. The selected solution had the least value of $v_{\infty, 2}$ in the set of feasible solutions.

The selected optimal design gene is presented in Table 5 . The design has a $C_{3, E}$ of $28.4 \mathrm{~km}^{2} / \mathrm{s}^{2}$ and a $v_{\infty, 2}$ of $2.33 \mathrm{~km} / \mathrm{s}$. A visualization of the designed heliocentric cruise is presented in Figure 14 . As seen in Table 5, the spacecraft will be launched from Earth on 2 October 2022, and will arrive at Mars on 7 June 2023 after cruising for a duration of 248 days. The spacecraft will arrive at Mars on an $R A A$ and $D A A$ of $80.8^{\circ}$ and $4.45^{\circ}$, respectively, in the Martian J2000 reference frame.

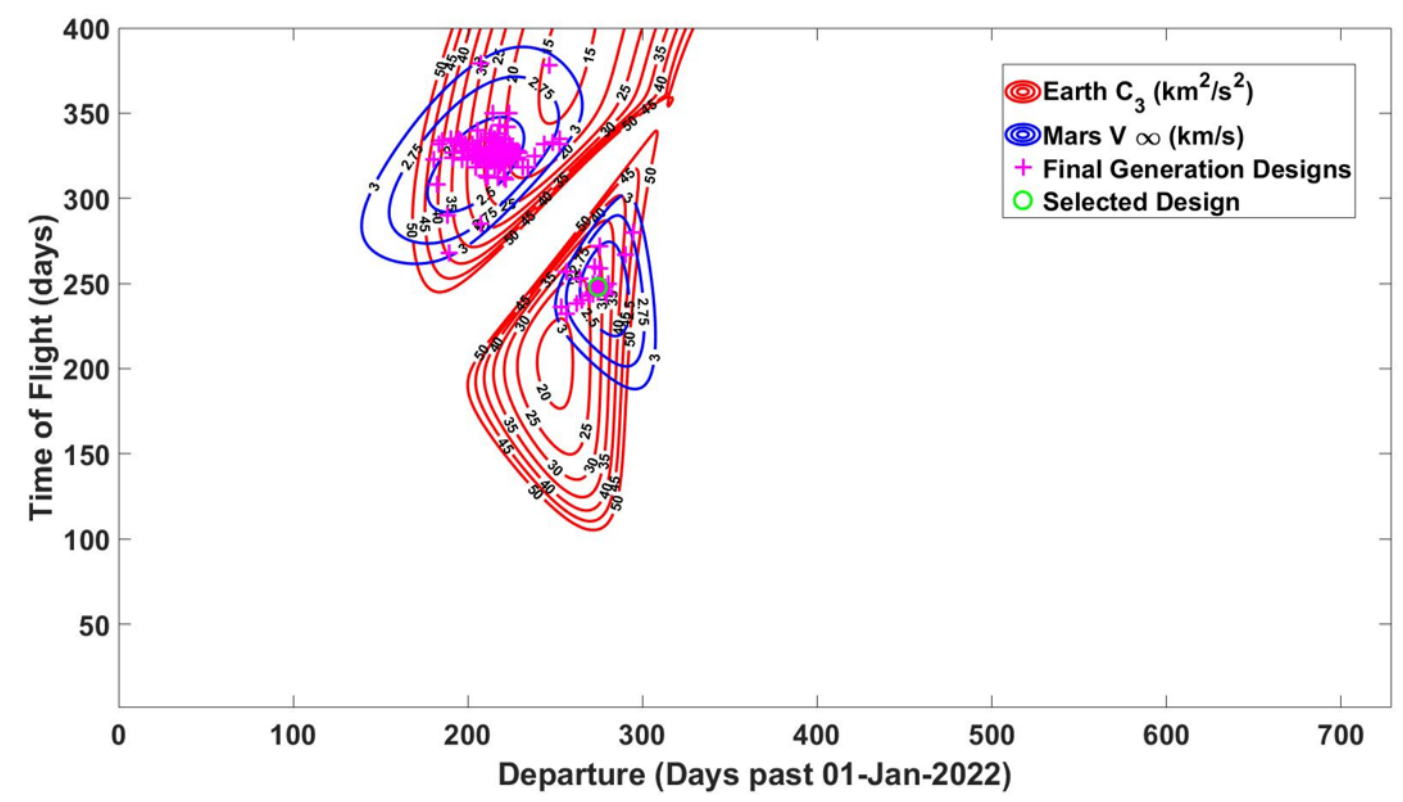

Figure 13. The figures of merit of the heliocentric cruise design problem shown on the Earth-Mars porkchop plot. 


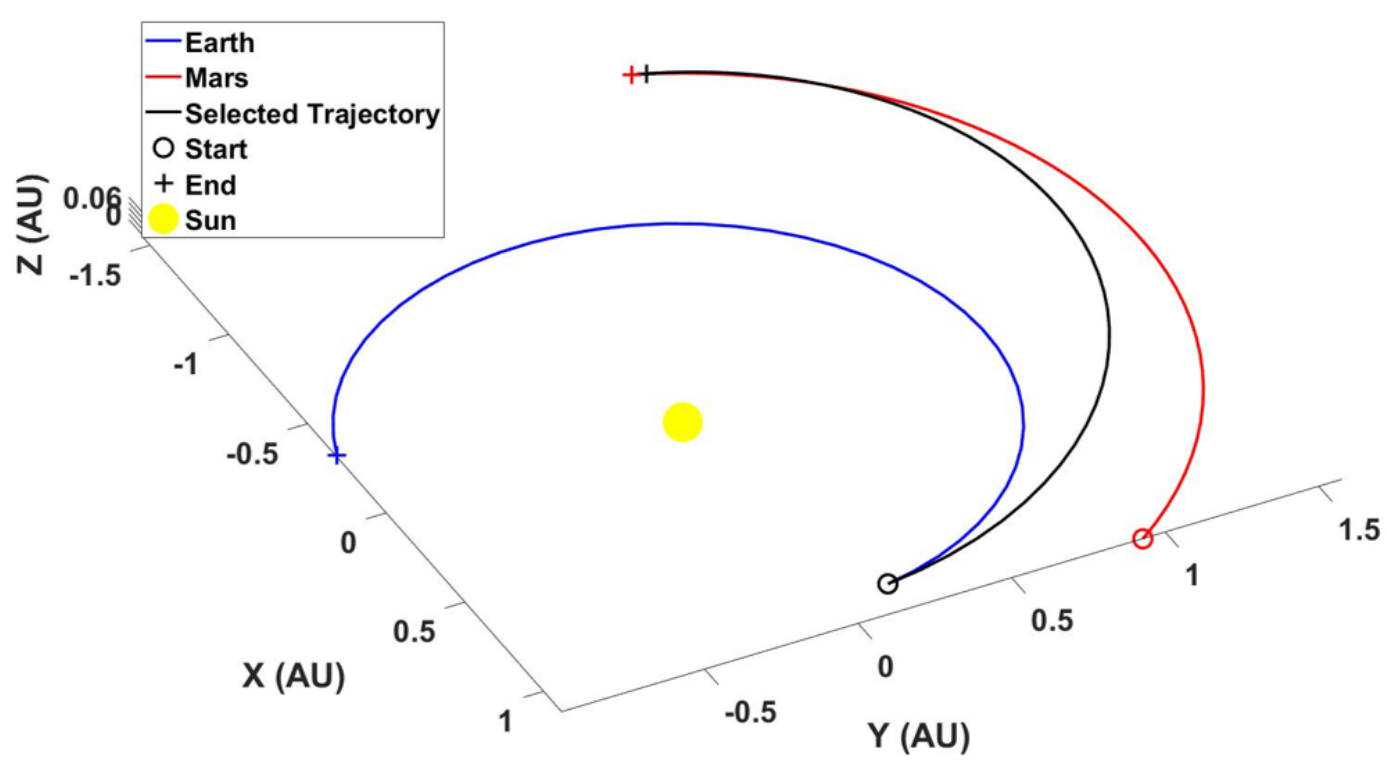

Figure 14. The designed heliocentric cruise solution showing the selected interplanetary trajectory between Earth and Mars.

Table 5. Design variables of the optimal heliocentric cruise design problem.

\begin{tabular}{|c|c|}
\hline Parameter & Value \\
\hline$D_{L}$ & 2 October 2022 \\
\hline$T o F$ & 248 days \\
\hline
\end{tabular}

\subsection{Dynamical Environment}

As mentioned above, the two-body dynamics is used to design the nominal mission concepts. The orbital elements of Phobos $[59,60]$ used in the current work are presented in Table 6 . The true anomaly at the start of simulations is obtained by offsetting the encounter true anomaly $f_{v}$ of Phobos $-2{ }^{\circ}$ and phasing the true spacecraft anomalies accordingly [52]. The maximum radius of Phobos was noted from a $32 \mathrm{k}$ triangular model of Phobos [61]. Additionally, to simulate the aerobraking maneuver at Mars, an altitude of $h_{A B}=150 \mathrm{~km}$ was used. The aerobraking altitude was selected based on the walk-in phase aerobraking altitude of the Mars Odyssey mission [62].

Table 6. Parameters used to model the orbit and spacecraft dynamics around Phobos.

\begin{tabular}{|l|c|}
\hline Parameter & Value \\
\hline Semimajor axis & $9376 \mathrm{~km}$ \\
\hline Eccentricity & 0.015 \\
\hline Inclination & $1.07^{\circ}$ \\
\hline RAAN & $208^{\circ}$ \\
\hline Argument of periapsis & $150^{\circ}$ \\
\hline Orbital period & 0.319 days \\
\hline Maximum radius & $14 \mathrm{~km}$ \\
\hline
\end{tabular}

\subsection{Co-Orbital Mission}

The MINLP problem in Equation (5) is solved using the GA optimizer with tailored selection, crossover, and mutation algorithms $[57,58]$. The input parameters to the co-orbital mission concept are presented in Table 7 . The weights were selected to equally emphasize the minimization of all three terms in Equation (5). The results of five GA optimization trials of the co-orbit optimization are presented in Figure 15. Each generation of a trial evaluated 500 co-orbit design genes. The minimum fitness identified by all GA trials was 0.733 . 
The final generations of all optimization trials identified 48 feasible solutions, whose figures of merit are presented in Figure 16. An optimal solution with the lowest value of $J_{O r b}$ and $\Delta v_{O C}$ was selected from this lot of solutions.
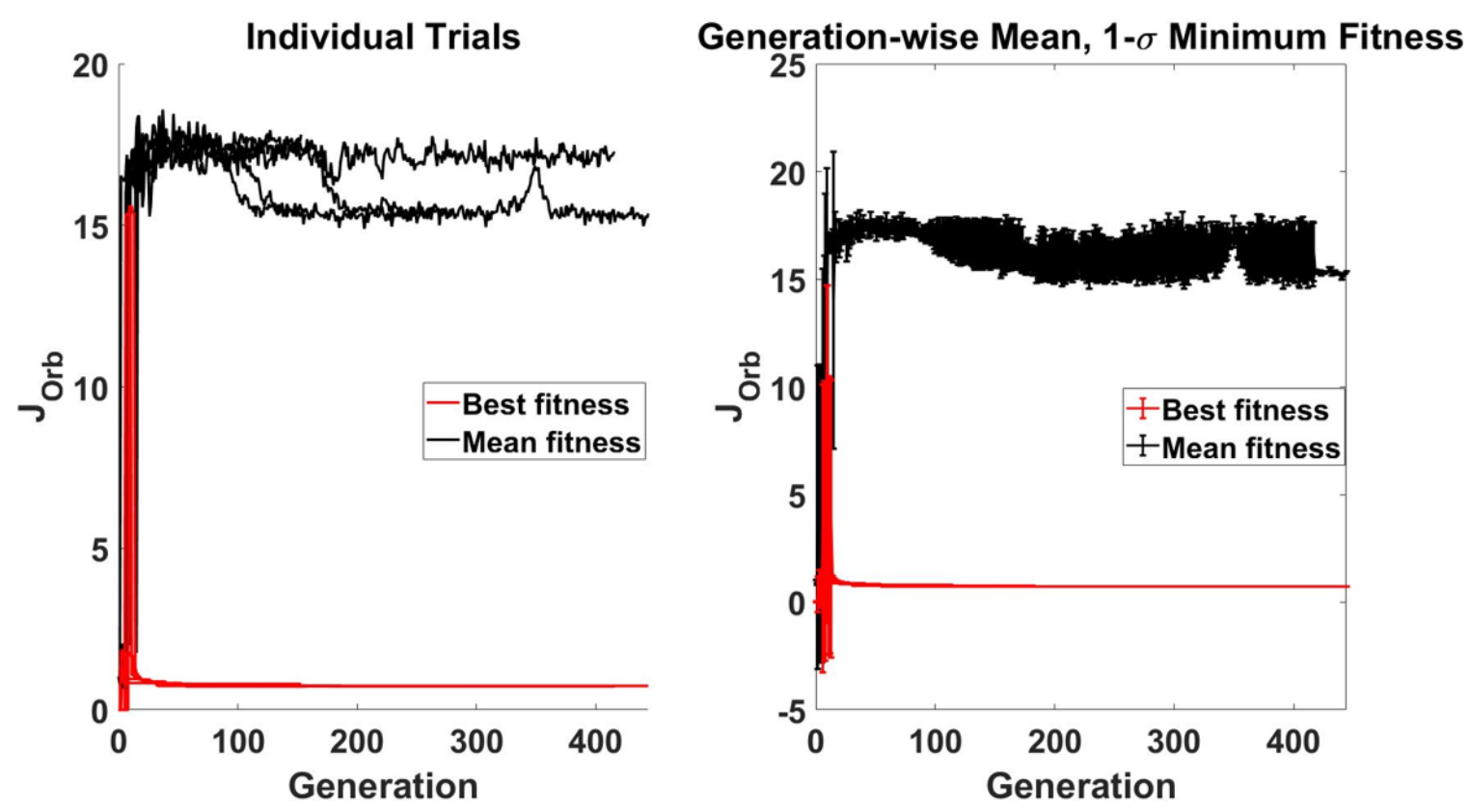

Figure 15. Variation of the co-orbital design fitness for the Perseus mission concept across different GA populations generations showing: the individual optimization trials (left); and their statistical distribution (right).
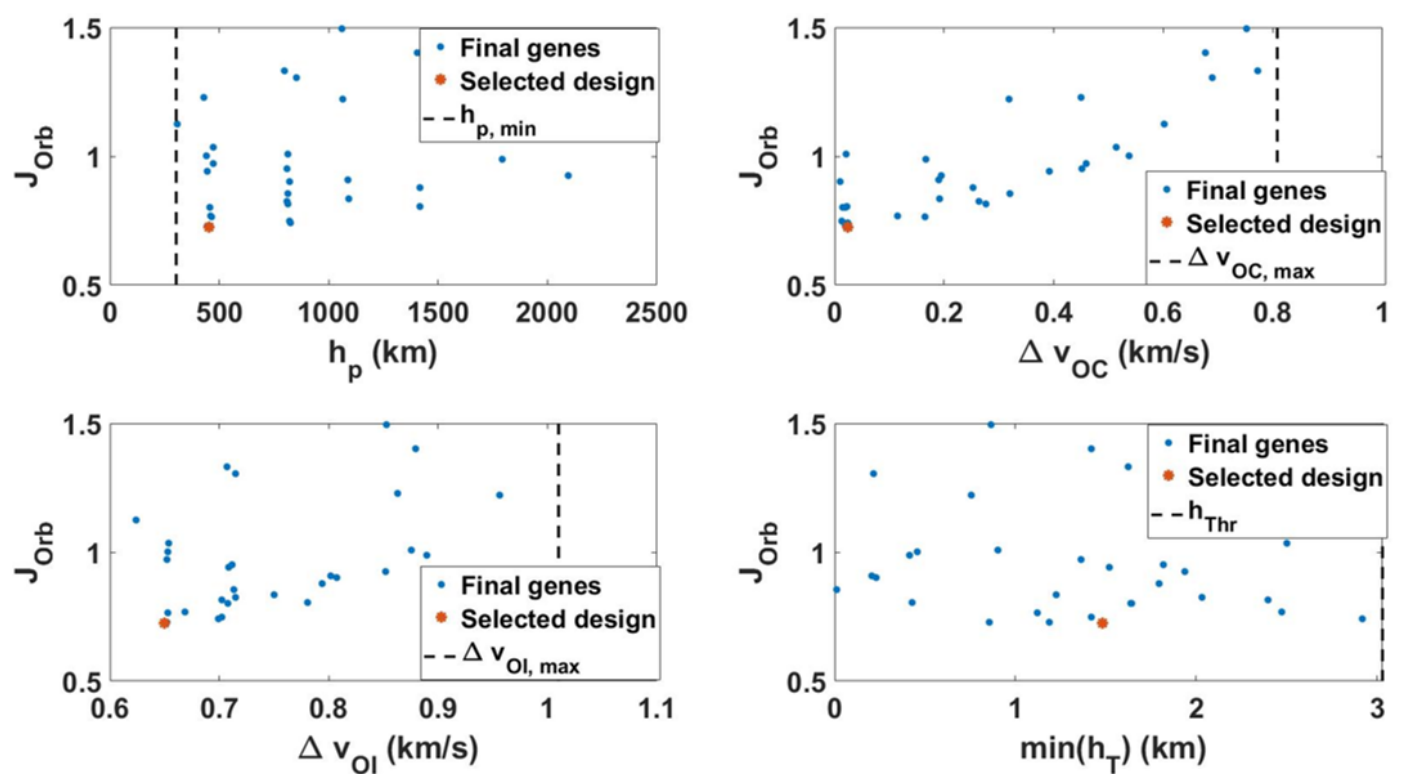

Figure 16. The figures of merit of the co-orbit design problem along with the selected optimal solution. 
Table 7. Parameters and bounds supplied as inputs to the co-orbital mission concept design.

\begin{tabular}{|c|c|}
\hline Parameter & Value \\
\hline$h_{p, \min }$ & $300 \mathrm{~km}$ \\
\hline$e_{0, \max }$ & 0.95 \\
\hline$\left[p_{\min }, p_{\max }\right]$ & {$[1,10]$} \\
\hline$\left[q_{\min }, q_{\max }\right]$ & {$[1,20]$} \\
\hline$\left[h_{\min }, h_{\max }\right]$ & {$[1,3]$} \\
\hline$w_{c, 1}$ & $1 \mathrm{~s}^{2} / \mathrm{km}$ \\
\hline$\left[w_{c, 2}, w_{c, 3}\right]$ & {$[1,1] \mathrm{s} / \mathrm{km}$} \\
\hline$\Delta v_{O I, \max }$ & $1 \mathrm{~km} / \mathrm{s}$ \\
\hline$\Delta v_{O C, \max }$ & $0.8 \mathrm{~km} / \mathrm{s}$ \\
\hline$h_{t h r}$ & $3 \mathrm{~km}$ \\
\hline
\end{tabular}

The design gene of the selected optimal solution is presented in Table 8 , which shows that the spacecraft will be in a 3:5 resonant co-orbit with Phobos, which allows the encounters to repeat after three orbits of Phobos or five orbits of the spacecraft (about one encounter per day). The periapsis altitude of the designed co-orbit was found to be $h_{p, C}=453 \mathrm{~km}$. The designed resonant co-orbit is presented in Figure 17.

Table 8. Design variables constituting the selected optimal co-orbit design gene.

\begin{tabular}{|c|c|}
\hline Parameter & Value \\
\hline$h_{p, 0}$ & $305 \mathrm{~km}$ \\
\hline$e_{0, \max }$ & 0.948 \\
\hline$p$ & 3 \\
\hline$q$ & 5 \\
\hline$h_{f}$ & $1.48 \mathrm{~km}$ \\
\hline$\theta_{x}$ & $145^{\circ}$ \\
\hline$\theta_{y}$ & $66.7^{\circ}$ \\
\hline$f_{v}$ & $215^{\circ}$ \\
\hline
\end{tabular}

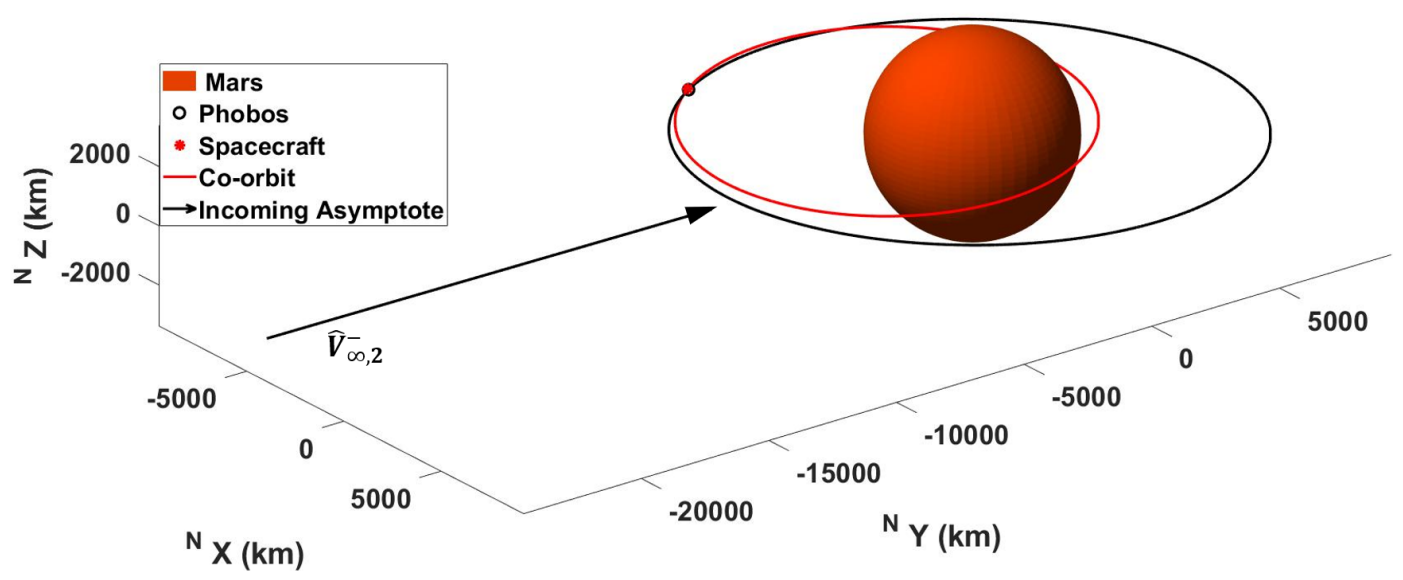

Figure 17. The designed resonant co-orbit of Perseus spacecraft during an encounter with Phobos.

\subsubsection{Orbit Insertion}

As noted in Table 8, the spacecraft will enter into an HEO with periapsis altitude of $305 \mathrm{~km}$ and an eccentricity of 0.948 before beginning its aerobraking maneuver. The orbit insertion cost associated with the three maneuvers, namely HEO capture, Walk-in, and Walk-out, was $\Delta v_{O I}=0.65 \mathrm{~km} / \mathrm{s}$. Additionally, the spacecraft required an orientation correction with a $\Delta v_{O I}=0.024 \mathrm{~km} / \mathrm{s}$, which leads to a net maneuver cost of $\Delta v_{\text {net }}=0.673 \mathrm{~km} / \mathrm{s}$. 


\subsubsection{Encounter Performance}

A simulated encounter of the Perseus spacecraft with respect to Phobos is presented in Figure 18. During this encounter the spacecraft will pass by Phobos at a closest altitude of $\min \left(h_{T}\right)=1.48 \mathrm{~km}$ which is also identical to its designed encounter altitude $h_{f}$. The designed co-orbit allows the spacecraft to spend $T_{E n c}=6.05 \mathrm{~min}$ inside the feasible region $\left(h_{T} \leq 100 \mathrm{~km}\right)$. During this time, the spacecraft will pass Phobos with relative velocities on the order of $0.55 \mathrm{~km} / \mathrm{s}$ as shown in Figure 19. Using the LMP-103s mono-propellant as the fuel (specific impulse of $240 \mathrm{~s}$ ), the fuel mass required is estimated [52] as $6.65 \mathrm{~kg}$. Therefore, this estimated fuel mass allows us a fuel margin of $55.6 \%$ in the allotted fuel mass of $15 \mathrm{~kg}$. This margin can be used as a fuel reserve for correction and station keeping maneuvers.

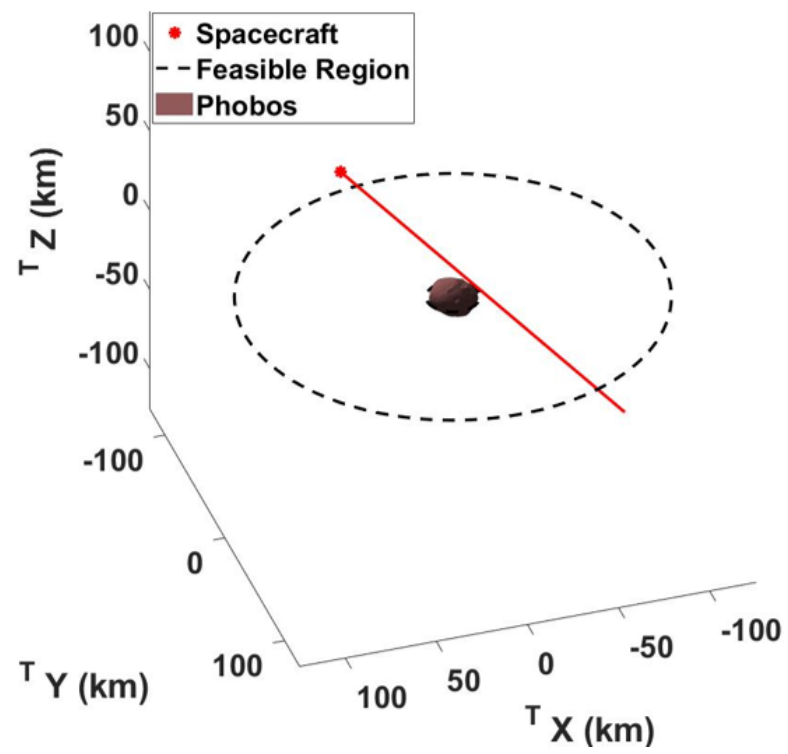

Figure 18. Visualization of spacecraft trajectory relative to Phobos during a simulated encounter.

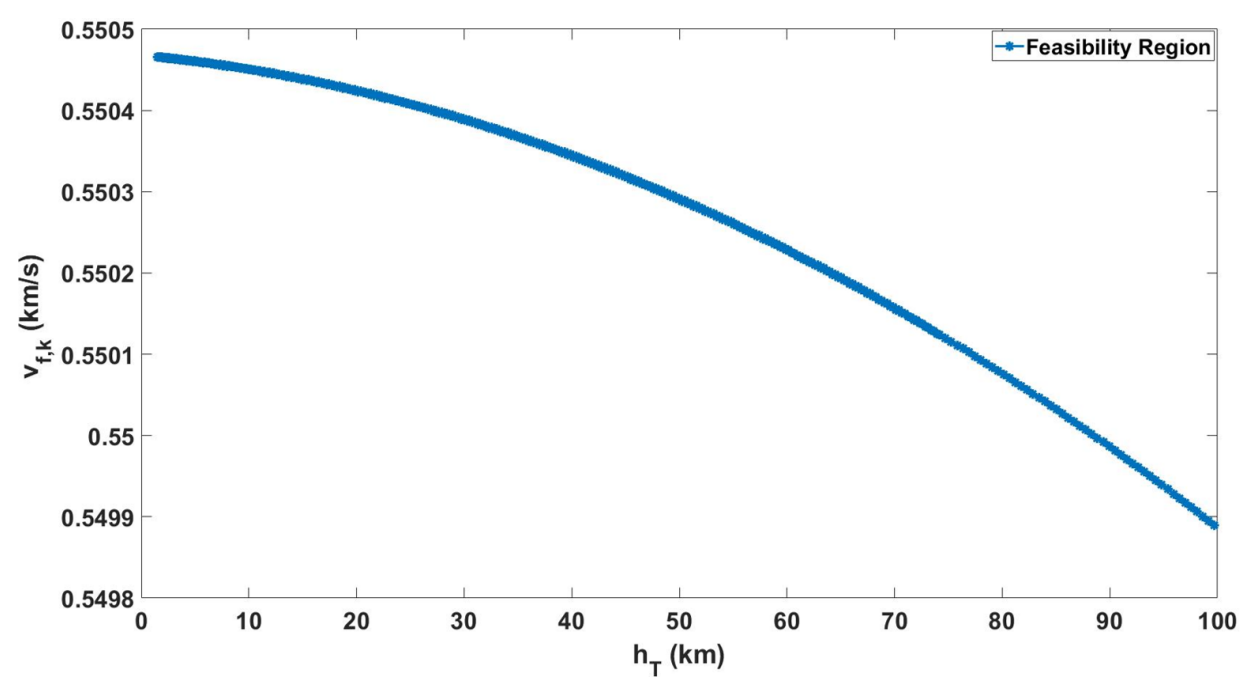

Figure 19. Relative velocities of the spacecraft with respect to Phobos inside the feasible region.

\subsection{Hyperbolic Mission}

The nonlinear programming problem in Equation (24) was solved using the proprietary GA solver in MATLAB [57]. The user input parameters to design the hyperbolic mission concept are presented in Table 9. The results of five GA optimization trials of the co-orbit optimization are presented in Figure 20. 
Each generation of a trial evaluated 100 co-orbit design genes. The minimum fitness identified by all GA trials was 1.693 .

Table 9. Parameters and bounds supplied as inputs to the hyperbolic mission concept design.

\begin{tabular}{|c|c|}
\hline Parameter & Value \\
\hline$\left[h_{\min }, h_{\max }\right]$ & {$[1,3] \mathrm{km}$} \\
\hline$w_{H}$ & $1 \mathrm{~s}^{2} / \mathrm{km}$ \\
\hline$\epsilon_{\max }$ & $10^{-5}$ \\
\hline$h_{t h r}$ & $3 \mathrm{~km}$ \\
\hline
\end{tabular}
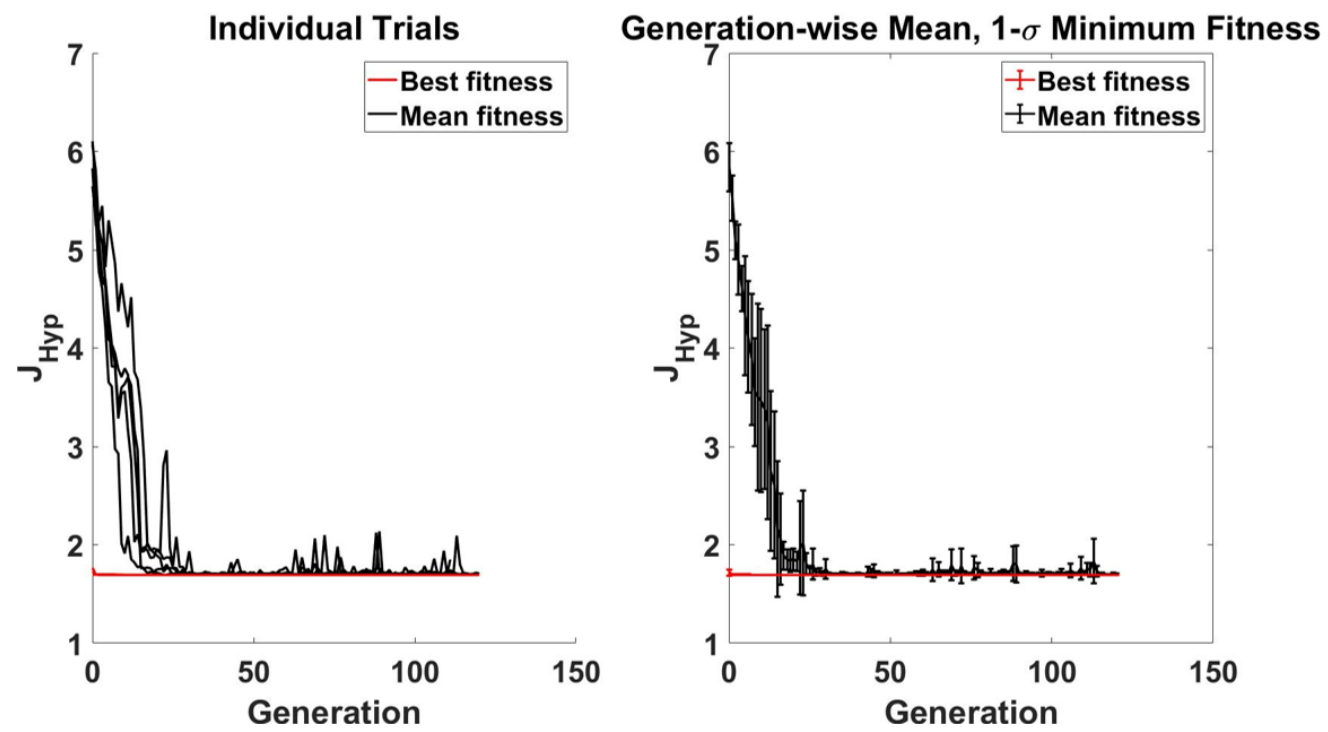

Figure 20. Variation of the hyperbolic design fitness for the Perseus mission concept across different GA populations generations showing: the individual optimization trials (left); and their statistical distribution (right).

The final generations of 440 feasible solutions whose figures of merit are presented in Figure 21. All constraints are shown to be satisfied. The design gene with the lowest root-solve error was selected as the optimal solution.
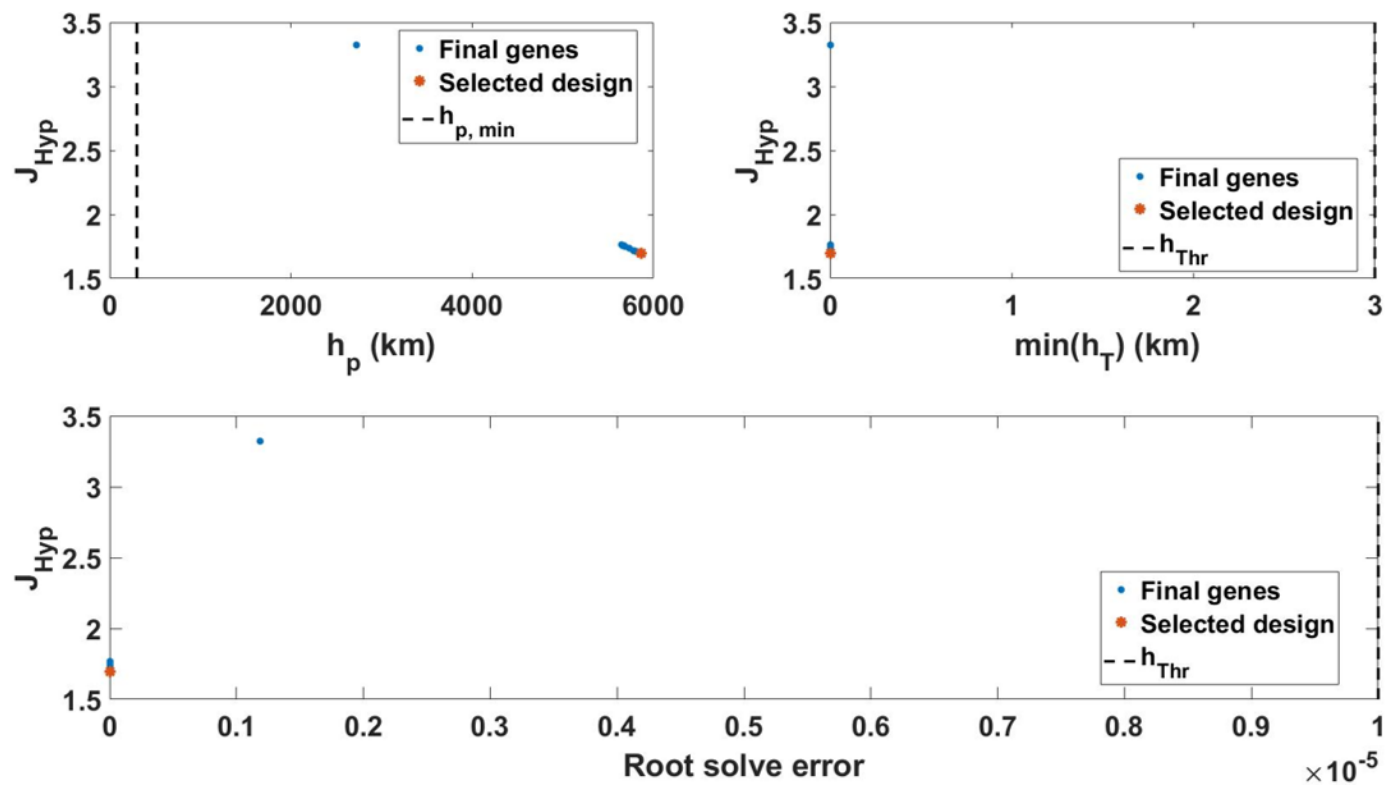

Figure 21. The figures of merit of the hyperbolic flyby design problem along with the selected optimal solution. 
The selected optimal solution is presented in Table 10. The selected gene has a martian periapsis altitude of $5834 \mathrm{~km}$ and a root solve error magnitude of $|\bar{\epsilon}|=1.74 \times 10^{-10}$. The designed hyperbolic trajectory is presented in Figure 22.

Table 10. Design variables constituting the selected optimal hyperbolic trajectory design gene.

\begin{tabular}{|c|c|}
\hline Parameter & Value \\
\hline$h_{f}$ & $2.99 \mathrm{~km}$ \\
\hline$\theta_{x}$ & $12.0^{\circ}$ \\
\hline$\theta_{y}$ & $16.0^{\circ}$ \\
\hline$f_{v}$ & $20.0^{\circ}$ \\
\hline
\end{tabular}

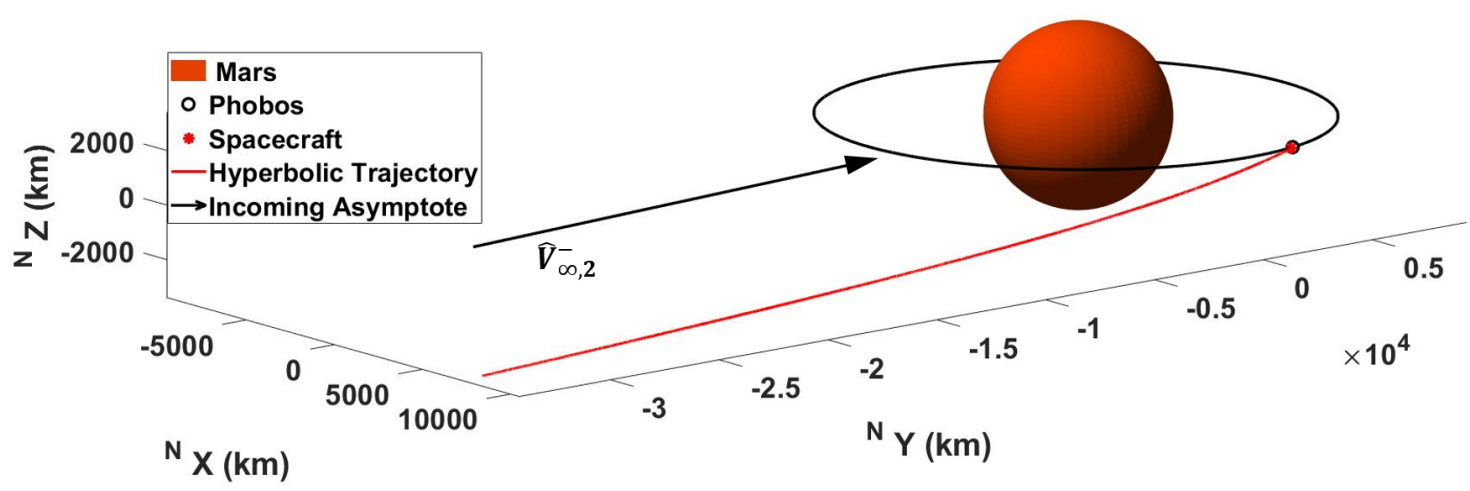

Figure 22. The designed hyperbolic trajectory of Perseus spacecraft during an encounter with Phobos.

\section{Encounter Performance}

During its encounter, the spacecraft will pass by Phobos at a closest altitude of $\min \left(h_{T}\right)=2.99 \mathrm{~km}$ which is also identical to its designed encounter altitude $h_{f}$. The designed trajectory allows the spacecraft to spend $T_{E n c}=2.22 \mathrm{~min}$ inside the feasible region $\left(h_{T} \leq 100 \mathrm{~km}\right)$. During this time, the spacecraft will pass Phobos with relative velocities on the order of $1.69 \mathrm{~km} / \mathrm{s}$, as shown in Figure 23 .

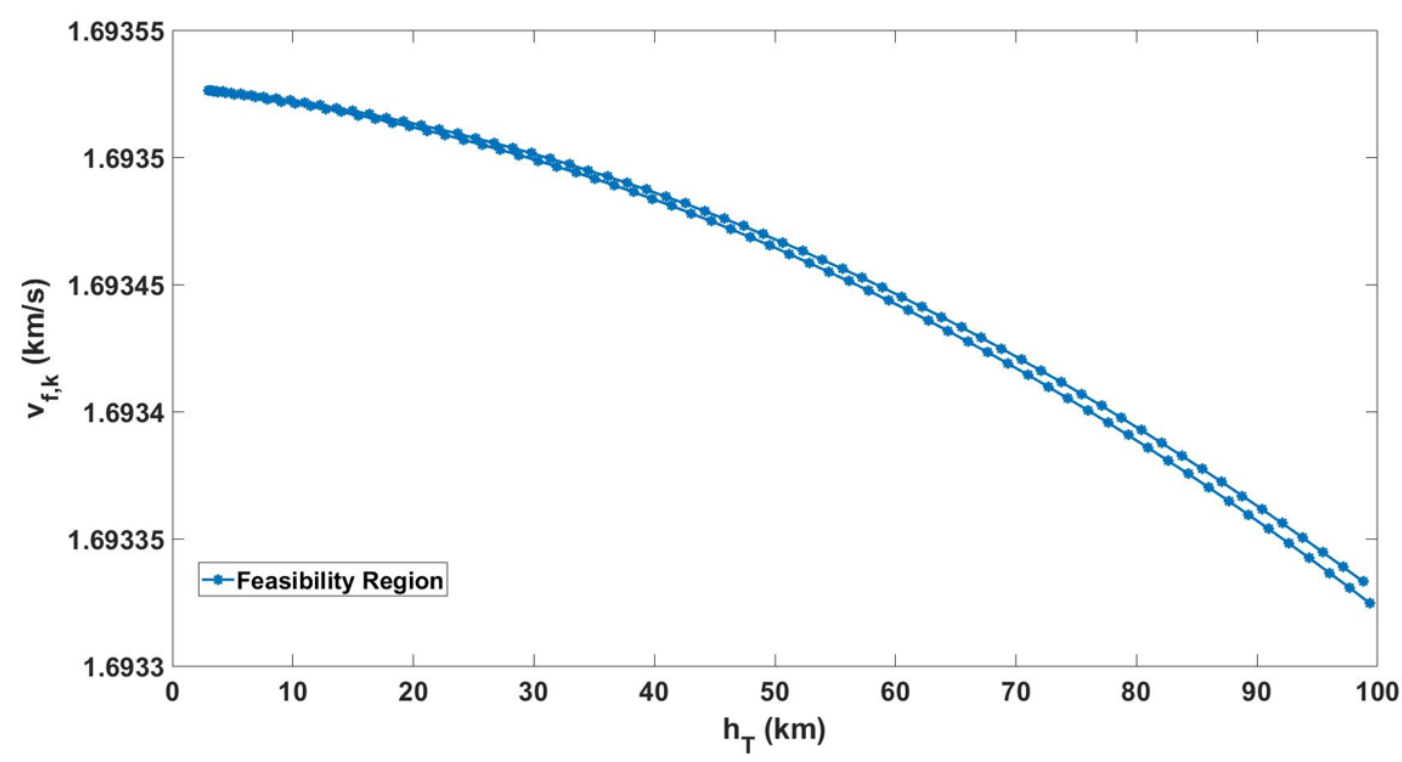

Figure 23. Relative velocities of the spacecraft with respect to Phobos during the hyperbolic encounter.

\subsection{Discussion}

This work revisits the importance of exploring the Martian moons. Here, we present the Perseus mission concept, which is a visual and thermal imaging mission to the Martian moon Phobos. This work explores the trajectory design of two mission concept alternatives for the Perseus mission 
using a design tree approach. The design tree's root node is the heliocentric cruise design problem, while the Phobos encounter configurations are arranged on the leaf nodes. This first concept is a co-orbital rendezvous with Phobos, while the second is a hyperbolic rendezvous. Both concepts are designed during the same launch window. The co-orbital mission concept is designed based on the nominal concept of operations of the mission. Here, the spacecraft will encounter Phobos nearly once per day in a 3:5 resonance. These encounters will occur for about $6.05 \mathrm{~min}$ with a relative velocity of $0.55 \mathrm{~km} / \mathrm{s}$. The deterministic maneuvers in the co-orbital mission concept have an estimated $\Delta v_{\text {net }}=0.673 \mathrm{~km} / \mathrm{s}$ to enter into the desired Martian orbit using an aeroassist from Mars. The estimated propellant consumption of these is about $6.65 \mathrm{~kg}$, which leaves a margin of $55.6 \%$ fuel on reserve. On the other hand, the hyperbolic mission concept is a descoped mission concept that allows us to explore Phobos using no deterministic maneuvers. However, the hyperbolic mission concept only allows a single pass of Phobos, where the spacecraft spends about 2.22 min inside the feasible region $\left(h_{T} \leq 100 \mathrm{~km}\right)$. During this encounter, the spacecraft passes by Phobos at higher relative velocities of about $1.69 \mathrm{~km} / \mathrm{s}$, which implies that the spacecraft requires higher slew rates to image Phobos.

The contributions of the current work to state-of-the-art spacecraft mission design research are described as follows. This work presents a holistic approach to design the trajectories of the Perseus mission concept to image the Martian moon Phobos. The mission requirements are formulated as optimization problems spanning a design tree. Additionally, we present algorithms to evaluate the encounter figures of merit of a specified design gene. While the derivation of co-orbital elements was presented in our previous work, this work derives the orbital elements of hyperbolic trajectories to rendezvous with planetary moons. The formulated design problems are then solved using evolutionary algorithms, and the performance of the selected optimal mission concepts is examined.

\section{Conclusions}

Mars exploration is at the forefront of human exploration. Several space agencies, including NASA and ESA, have identified Mars as a long-term destination for human exploration. Scientifically, Phobos is along this strategic path, because, ultimately, it is tied to our understanding of the origin of Mars, which itself is a mystery that may hold the key to the origin of Earth. In this paper, we outline a science reconnaissance mission to Phobos using a 27 U CubeSat mission concept called Perseus. Meeting the science goals of Perseus shall lead to a global-scale characterization of Phobos geology and provide insight into the dynamically changing surface environment. Insight into the dynamical surface environment of Phobos can help to derisk future surface missions and plans to setup human transit base on Phobos. The visible images shall reveal, more consistently and globally than before, how the grooves cross-cut each other; how they vary photometrically; and how grooves correlate with thermal inertia, that is representative of the particle size or matrix. Detail characterization of the surface of Phobos can provide greater insight into the origin of Phobos, particularly if it was formed from Martian debris or is a captured asteroid. The Perseus mission is, nominally, a co-orbiter mission that images Phobos upon regularly timed encounters during its Martian orbits. While the co-orbits allow repeated encounters, we also examine a descoped mission concept of exploring Phobos through a direct hyperbolic flyby. While such a flyby allows a single imaging opportunity at higher relative speeds, it also enables exploring the moon with reduced fuel requirements and, consequently, reduced mission costs. The presented concepts are still at a very early stage in the design process. However, our design studies use several high-TRL subsystems as baseline components.

While the current work explores the theoretical mission concept design, future work on the Perseus mission concept will focus on integration and testing of the subsystems of the Perseus spacecraft. Specifically, the performance of the propulsion and ADCS subsystems will be evaluated to verify if these can achieve their required performance in practice. Similarly, the payload capability will be tested to verify if visible and thermal cameras can generate images of the required resolution in practice. These tests will enable us to identify the final mission concept and allow us to demonstrate the utility of the CubeSats in performing planetary moon reconnaissance. 
Author Contributions: The contributions to this article are the following. Conceptualization, J.T. and E.A.; methodology, G.D., N.K., J.U., S.I., A.C. (Akshay Choudhari), M.H.-M., A.C. (Aman Chandra); software, R.t.N. and J.U.; validation, R.t.N. and S.S.; formal analysis, J.U. and R.t.N.; investigation, J.U. and R.t.N.; resources, J.T.; data curation, R.t.N. and J.U.; writing-original draft preparation, G.D., N.K., J.U., S.I., A.C. (Akshay Choudhari), M.H.-M., A.C. (Aman Chandra) and J.T.; writing-review and editing, R.t.N., J.T. and S.S.; visualization, A.C. (Akshay Choudhari), R.t.N., J.T.; supervision, J.T. and E.A.; project administration, J.T.; funding acquisition, J.T. All authors have read and agreed to the published version of the manuscript.

Funding: This research was funded in part by National Aeronautics and Space Administration, grant number 80NSSC19M0197 and NASA JPL SURP Funding.

Conflicts of Interest: The authors declare no conflict of interest.

\section{References}

1. Staehle, R.; Blaney, D.; Hemmati, H.; Jones, D.; Anderson, B.; Betts, B.; Chow, C.; Friedman, L.; Klesh, A.; Liewer, P.; et al. Interplanetary CubeSats: Opening the Solar System to a Broad Community at Lower Cost. J. Small Satell. 2013, 2, 161-186.

2. Schoolcraft, J.; Klesh, A.; Werne, T. MarCO: Interplanetary Mission Development On a CubeSat Scale. In Proceedings of the AIAA SpaceOps 2016 Conference, SpaceOps Conferences, Daejeon, Korea, 16-20 May 2016; p. 2491.

3. Hodges, R.; Hoppe, D.J.; Radway, M.J.; Chahat, N.E. Novel Deployable Reflectarray Antennas for CubeSat Communications. In Proceedings of the 2015 IEEE MTT-S International Microwave Symposium, Phoenix, AZ, USA, 17-22 May 2015.

4. Asphaug, E. Rise and Fall of the Martian Moons. Nat. Geosci. 2016, 9, 568-569. [CrossRef]

5. Ball, A.J.; Price, M.E.; Walker, R.J.; Dando, G.C.; Wells, N.S.; Zarnecki, J.C. Mars Phobos and Deimos Survey (M-PADS)_A Martian Moons Orbiter and Phobos Lander. Adv. Space Res. 2009, 43, 120-127. [CrossRef]

6. Duxbury, T.C. Spacecraft Exploration of Phobos and Deimos. Planet. Space Sci. 2014, 102, 9-17. [CrossRef]

7. Murchie, S.L. The Scientific Rationale for Robotic Exploration of Phobos and Deimos; Applied Physics Laboratory, Johns Hopkins University: Laurel, MD, USA, 2009.

8. Nayak, M.; Asphaug, E. Sesquinary catenae on the Martian satellite Phobos from reaccretion of escaping ejecta. Nat. Commun. 2016, 7, 12591. [CrossRef]

9. National Research Council. Vision and Voyages for Planetary Science in the Decade 2013-2022; The National Academies Press: Washington, DC, USA, 2011.

10. National Aeronautics and Space Administration. NASA's Journey to Mars; NASA Head Quarters: Washington, DC, USA, 2015.

11. Waldron, R.D. A Survey of Resource Utilization Processes for Mars and Its Moons. In Proceedings of the 14th SSI/Princeton Conference on Space Manufacturing, Space Studies Institute, Princeton, NJ, USA, 6-9 May 1999; pp. 53-63.

12. Muscatello, A.C.; Mueller, R.; Sanders, G.B.; Larson, W.E. Phobos and Deimos Sample Collection Missions for Science and ISRU. In Proceedings of the Lunar and Planetary Science Conference (LPSC), Houston, TX, USA, 19-23 March 2012; p. 4296.

13. Miyamoto, H. Japanese Mission of the Two Moons of Mars with Sample Return from Phobos; Mars Exploration Program Analysis Group (MEPAG): Pasadena, CA, USA, 2016.

14. Campagnola, S. Mission Analysis for the Martian Moons Explorer (MMX) mission. In Proceedings of the International Astronautica Congress, Guadalajara, Mexico, 26-30 September 2016; pp. 1-8.

15. Klesh, A. INSPIRE and Beyond-Deep Space CubeSats at JPL. NASA JPL Tech Report. In Proceedings of the 2015 CubeSat Developers Workshop, San Luis Obispo, CA, USA, 27-29 April 2015.

16. Taylor, C.; Shao, A.; Armade, N. Hummingbird: Versatile Interplanetary Mission Architecture. In Proceedings of the Interplanetary Small Satellite Conference, Pasadena, CA, USA, 20-21 June 2013.

17. Hardgrove, C.; Bell, J.; Thangavelautham, J.; Klesh, A.; Starr, R.; Colaprete, T.; Robinson, M.; Drake, D.; Johnson, E.; Genova, A.; et al. The Lunar Polar Hydrogen Mapper (LunaH-Map) mission: Mapping hydrogen distributions in permanently shadowed regions of the Moon's south pole. In Proceedings of the 46th Lunar and Planetary Science Conference, Houston, TX, USA, 16-20 March 2015. 
18. Clark, P.; Malphrus, B.; Brown, K. Lunar Ice Cube Mission: Determining Lunar Water Dynamics with a First Generation Deep Space CubeSat. In Proceedings of the 47th Lunar and Planetary Science Conference, Houston, TX, USA, 21-25 March 2016.

19. Topputo, F.; Massari, M.; Bigg, J.; Tos, D.A.D.; Ceccherini, S.; Mani, K.V.; Franzese, V.; Cervone, A.; Sundaramoorthy, P.; Mestry, S.; et al. LUMIO: Lunar Meteoroid Impact Observer. In Proceedings of the ICubeSat Conference 2017, Cambridge, UK, 30-31 May 2017.

20. McNutt, L.; Johnson, L.; Kahn, P.; Castillo-Rogez, J.; Frick, A. Near-Earth Asteroid (NEA) Scout. In Proceedings of the AIAA SPACE Conference, San Diego, CA, USA, 4-7 August 2014.

21. Segret, B.; Vannitsen, J.; Agnan, M. BIRDY: BIRDY: An interplanetary CubeSat to collect radiation data on the way to Mars and back. In Proceedings of the European Planetary Science Congress 2014, Cascais, Portugal, 7-12 September 2014.

22. Chanover, N.; Murphy, J.; Rankin, K.; Stochaj, S.; Thelen, A. A Europa CubeSat Concept Study for Measuring Europa's Atmosphere. In Proceedings of the Small Sat Conference, Logan, UT, USA, 6-11 August 2016.

23. Thangavelautham, J.; Rhoden, A.; Drew, J. The Opportunities and Challenges of GN\&C on a Europa CubeSat to Search for Plumes, Surface Fractures and Landing Sites. In Proceedings of the 40th AAS Guidance and Control Conference, Breckenridge, CO, USA, 2-8 February 2017.

24. Rao, S.S. Engineering Optimization: Theory and Practice; John Wiley \& Sons: Hoboken, NJ, USA, 2019.

25. Conn, A.R.; Gould, N.I.M.; Toint, P.L. A Globally Convergent Augmented Lagrangian Algorithm for Optimization with General Constraints and Simple Bounds. SIAM J. Numer. Anal. 1991, 28, 545-572. [CrossRef]

26. Lohn, J.D.; Linden, D.S.; Hornby, G.S.; Kraus, W.F. Evolutionary design of an X-band antenna for NASA's space technology 5 mission. In Proceedings of the IEEE Antennas and Propagation Society Symposium, Monterey, CA, USA, 20-25 June 2004; Volume 3, pp. 2313-2316.

27. Hartmann, J.W.; Coverstone-Carroll, V.L.; Williams, S.N. Optimal interplanetary spacecraft trajectories via a Pareto genetic algorithm. J. Astronaut. Sci. 1998, 46, 267-282. [CrossRef]

28. Nallapu, R.; Thangavelautham, J. Towards End-To-End Design of Spacecraft Swarms for Small-Body Reconnaissance. In Proceedings of the International Astronautical Congress, IAC, International Astronautical Federation, IAF, Washington, DC, USA, 21-25 October 2019.

29. Nallapu, R.; Thangavelautham, J. Automated design architectures for co-orbiting spacecraft swarms for planetary moon mapping. Adv. Space Res. 2020. [CrossRef]

30. Nallapu, R.; Thangavelautham, J. Design and Sensitivity Analysis of Spacecraft Swarms for Planetary Moon Reconnaissance through Co-orbits. Acta Astronaut. 2020. [CrossRef]

31. Nallapu, R.; Thangavelautham, J. Design of Spacecraft Swarm Flybys for Planetary Moon Exploration. In Proceedings of the AIAA Scitech 2020 Forum, Orlando, FL, USA, 6-10 January 2020; p. 0954.

32. Delbo, M.; Libourel, G.; Wilkerson, J.; Murdoch, N.; Michel, P.; Ramesh, K.T.; Ganino, C.; Verati, C.; Marchi, S. Thermal fatigue as the origin of regolith on small asteroids. Nature 2014, 508, 233-236. [CrossRef]

33. Harris, A.W.; Drube, L. Asteroid Thermal Inertia Estimates from Remote Infrared Observations: The Effects of Surface Roughness and Rotation Rate. Astrophys. J. 2020, 901, 140. [CrossRef]

34. Salvail, J.R.; Fanale, F.P. Near-surface ice on Mercury and the Moon: A topographic thermal model. Icarus 1994, 111, 441-455. [CrossRef]

35. Paige, D.A.; Siegler, M.A.; Harmon, J.K.; Neumann, G.A.; Mazarico, E.M.; Smith, D.E.; Zuber, M.T.; Harju, E.; Delitsky, M.L.; Solomon, S.C. Thermal stability of volatiles in the north polar region of Mercury. Science 2013, 339, 300-303. [CrossRef] [PubMed]

36. Siegler, M.A.; Miller, R.S.; Keane, J.T.; Laneuville, M.; Paige, D.A.; Matsuyama, I.; Lawrence, D.J.; Crotts, A.; Poston, M.J. Lunar true polar wander inferred from polar hydrogen. Nature 2016, 531, 480-484. [CrossRef]

37. Rambaux, N.; Castillo-Rogez, J.C.; Le Maistre, S.; Rosenblatt, P. Rotational motion of Phobos. Astron. Astrophys. 2012, 548, A14. [CrossRef]

38. Ballouz, R.L.; Baresi, N.; Crites, S.T.; Kawakatsu, Y.; Fujimoto, M. Surface refreshing of Martian moon Phobos by orbital eccentricity-driven grain motion. Nat. Geosci. 2019, 12, 229-234. [CrossRef]

39. Walsh, K.J.; Jawin, E.R.; Ballouz, R.L.; Barnouin, O.S.; Bierhaus, E.B.; Connolly, H.C.; Molaro, J.L.; McCoy, T.J.; Delbo, M.; Hartzell, C.M.; et al. Craters, boulders and regolith of (101955) Bennu indicative of an old and dynamic surface. Nat. Geosci. 2019, 12, 242-246. [CrossRef] 
40. Bottke, W.F.; Vokrouhlický, D.; Ballouz, R.L.; Barnouin, O.S.; Connolly, H.C., Jr.; Elder, C.; Marchi, S.; McCoy, T.J.; Michel, P.; Nolan, M.C.; et al. Interpreting the Cratering Histories of Bennu, Ryugu, and Other Spacecraft-explored Asteroids. Astron. J. 2020, 160, 14. [CrossRef]

41. Hopkins, J.; Pratt, W. Comparison of Deimos and Phobos as Destinations for Human Exploration and Identification of Preferred Landing Sites. In Proceedings of the AIAA Space Conference, Long Beach, CA, USA, 27-29 September 2011.

42. Nallapu, R.; Schwartz, S.R.; Asphaug, E.; Thangavelautham, J. Robust Spin Control Design for the AOSAT+ Mission Concept. IEEE J. Miniaturization Air Space Syst. 2020, 1, 10-31. [CrossRef]

43. Hernandez, V.; Gankidi, P.; Chandra, A.; Miller, A.; Scowen, P.; Barnaby, H.; Adamson, E.; Asphaug, E.; Thangavelautham, J. SWIMSat: Space Weather and Meteor Impact Monitoring using a Low-Cost 6U CubeSat. In Proceedings of the Small Satellite Conference 2016, Logan, UT, USA, 6-11 August 2016.

44. Veto, M.S.; Christensen, P.R.; Spencer, D.A. The thermal-camera for exploration, science, and imaging spacecraft. In Proceedings of the Lunar and Planetary Science Conference, Houston, TX, USA, 21-25 March 2016; p. 2877.

45. Wen, Y.; Rauscher, B.J.; Baker, R.G.; Clampin, M.C.; Fochie, P.; Heap, S.R.; Hilton, G.; Jorden, P.; Linder, D.; Mott, B.; et al. Individual photon counting using e2v L3 CCDs for low background astronomical spectroscopy. In High Energy, Optical, and Infrared Detectors for Astronomy II. Int. Soc. Opt. Photonics 2006, 6276, 62761H.

46. Blake, D.; Vaniman, D.; Achilles, C.; Anderson, R.; Bish, D.; Bristow, T.; Chen, C.; Chipera, S.; Crisp, J.; Des Marais, D.; et al. Characterization and calibration of the CheMin mineralogical instrument on Mars Science Laboratory. Space Sci. Rev. 2012, 170, 341-399. [CrossRef]

47. Leomanni, M.; Garulli, A.; Giannitrapani, A.; Scortecci, F. Propulsion options for very low Earth orbit microsatellites. Acta Astronaut. 2017, 133, 444-454. [CrossRef]

48. Zeitlin, C.; Hassler, D.M.; Cucinotta, F.A.; Ehresmann, B.; Wimmer-Schweingruber, R.F.; Brinza, D.E.; Kang, S.; Weigle, G.; Bottcher, S.; Bohm, E.; et al. Measurements of Energetic Particle Radiation in Transit to Mars on the Mars Science Laboratory. Science 2013, 340, 1080-1084. [CrossRef] [PubMed]

49. Chien, S.; Doubleday, J.; Ortega, K.; Tran, D.; Bellardo, J.; Williams, A.; Piug-Suari, J.; Crum, G.; Flatley, T. Onboard autonomy and ground operations automation for the Intelligent Payload Experiment (IPEX) CubeSat Mission. In Proceedings of the International Symposium on Artificial Intelligence, Robotics and Automation in Space, Turin, Italy, 4-6 September 2012.

50. Naasz, B.; Eepoel, J.V.; Queen, S.; Southward, C.M.; Hannah, J. Flight results from the HST SM4 Relative Navigation Sensor system. In Proceedings of the AAS Guidance Navigation and Control Conference, Breckenridge, CO, USA, 5-10 February 2010.

51. Imbriale, W.A.; Imbriale, W.A. Large Antennas of the Deep Space Network; Wiley-Interscience: Hoboken, NJ, USA, 2003; p. 302.

52. David, A. Vallado, Fundamentals of Astrodynamics and Applications, 4th ed.; Microcosm Press: Hawthorne, CA, USA, 2013.

53. Spencer, D.A.; Tolson, R. Aerobraking cost and risk decisions. J. Spacecr. Rocket. 2007, 44, $1285-1293$. [CrossRef]

54. Schaub, H.; Junkins, J.L. Analytical Mechanics of Space Systems, 4th ed.; AIAA Education Series; AIAA: Reston, VA, USA, 2018. [CrossRef]

55. Conn, A.R.; Gould, N.I.; Toint, P.L. Trust Region Methods; Society for Industrial and Applied Mathematics; SIAM: Philadelphia, PA, USA, 2000.

56. Russell, R.P. On the solution to every Lambert problem. Celest. Mech. Dyn. Astron. 2019, 131, 50. [CrossRef]

57. Deb, K. An efficient constraint handling method for genetic algorithms. Comput. Methods Appl. Mech. Eng. 2000, 186, 311-338. [CrossRef]

58. Deep, K.; Singh, K.P.; Kansal, M.L.; Mohan, C. A real coded genetic algorithm for solving integer and mixed integer optimization problems. Appl. Math. Comput. 2009, 212, 505-518. [CrossRef]

59. Murray, C.D.; Dermott, S.F. Solar System Dynamics; Cambridge University Press: Cambridge, NY, USA, 1999.

60. Giorgini, J.D. Status of the JPL Horizons Ephemeris System. In Proceedings of the IAU General Assembly 22, Honolulu, HI, USA, 3-14 August 2015.

61. Frieger, G. Space Frieger Shape Model Catalogue. 2018. Available online: https://space.frieger.com/ asteroids / (accessed on 17 October 2020). 
62. Smith, J.C., Jr.; Bell, J.L. 2001 Mars Odyssey Aerobraking. J. Spacecr. Rocket. 2005, 42, 406-415. [CrossRef]

Publisher's Note: MDPI stays neutral with regard to jurisdictional claims in published maps and institutional affiliations.

(C) 2020 by the authors. Licensee MDPI, Basel, Switzerland. This article is an open access article distributed under the terms and conditions of the Creative Commons Attribution (CC BY) license (http://creativecommons.org/licenses/by/4.0/). 